This item was submitted to Loughborough's Research Repository by the author.

Items in Figshare are protected by copyright, with all rights reserved, unless otherwise indicated.

\title{
Hybrid LES-RANS study of an effusion cooling array with circular holes
}

\section{PLEASE CITE THE PUBLISHED VERSION}

https://doi.org/10.1016/j.ijheatfluidflow.2018.09.014

\section{PUBLISHER}

(C) Elsevier, Inc.

\section{VERSION}

AM (Accepted Manuscript)

\section{PUBLISHER STATEMENT}

This paper was accepted for publication in the journal International Journal of Heat and Fluid Flow and the definitive published version is available at https://doi.org/10.1016/j.ijheatfluidflow.2018.09.014.

\section{LICENCE}

CC BY-NC-ND 4.0

\section{REPOSITORY RECORD}

Chen, Xiao-Sheng, and Hao Xia. 2019. "Hybrid LES-RANS Study of an Effusion Cooling Array with Circular Holes". figshare. https://hdl.handle.net/2134/36882. 


\title{
Hybrid LES-RANS study of an effusion cooling array with circular holes
}

\author{
X. Chen and H. Xia \\ Department of Aeronautical and Automotive Engineering, Loughborough University, Loughborough, United Kingdom
}

\begin{abstract}
In this paper, a multi-row effusion cooling configuration with scaled gas turbine combustor conditions is studied numerically. The distribution of the coolant film is examined by surface adiabatic cooling effectiveness (ACE). Simulation results have shown that the accuracy of cooling effectiveness prediction is closely related to the resolution of turbulent flow structures involved in hot-cold flow mixing, especially those close to the plate surface. The formation of the coolant film in the streamwise direction is investigated. It is shown that the plate surface directly downstream the coolant holes are covered well by the coolant jets, while surface regions in between the two columns of the coolant holes could not be protected until the coolant film is developed sufficiently in the spanwise direction in the downstream region. More detailed study has also been carried out to study the time-averaged and time-dependent flow field. The relation between the turbulent flow structures and coolant film distribution are also examined. The Kelvin-Helmholtz instability in the upper and lower coolant jet shear layer, is found to have the same frequency of around $8000 \mathrm{~Hz}$, and is independent of the coolant hole position. Additionally, it is suggested by the spectral coherence analysis that those unsteady flow structures from the lower shear layer are closely related to the near-wall flow temperature, and such effect is also independent of the coolant hole position.
\end{abstract}

Keywords: hybrid LES-RANS, multi-row effusion cooling, inflow turbulence, adiabatic cooling effectiveness

\section{Introduction}

The combustion chamber of a modern gas turbine engine is a hostile environment in which the combustor liner is exposed to the extremely high temperature gas. Effusion cooling is a commonly used technology to protect the combustor liner from being melted down by the hot gas. By injecting solid surface to isolate it from the high temperature gas. Increasing the coverage of the coolant film improves the cooling effect. However, simply increasing the amount of coolant will reduce the efficiency of jet engine components as the coolant air is often routed from the compressor, hence decreasing the propulsive mass flow. Good understanding of the effusion cooling mechanism is critical for improving 10 the cooling performance with minimum coolant usage. Moreover, the flow inside a combustor chamber is highly unsteady with injection, swirls and vortices, as well as chemical reaction of fuel-air mixture. It is therefore important to understand the effects of such highly unsteady flow on the formation of coolant film. 
There are a wide range of studies carried out to investigate the performance of the coolant film both experimentally and numerically. A series of experiments were carried out by Sinha and others [1-4] on a configuration of a single-row of $30^{\circ}$-inclined coolant holes. Numerical simulations of this configuration using the Reynolds-averaged Navier-Stokes (RANS) approach have been performed by many groups as well [5 7]. Although these RANS studies were generally able to reproduce the time-averaged flow quantities with reasonable accuracy, a time-dependent approach such as Large-Eddy Simulation (LES) will offer great advantages in resolving the unsteady flow structures as the mixing of the coolant and mainstream is dominated by secondary flows and vortex interactions. Several groups have conducted LES studies [8 10] on single-row configurations and obtained good agreement with experimental data. Ziefle and Kleiser [11] carried out an LES study with inflow turbulence added to the mainstream as inlet boundary conditions. Differences in the cooling effectiveness distribution were found compared to those without inflow turbulence.

Resolving the flow in each of the hundreds or thousands of holes in a full effusion cooling array would be very expensive. As long as only a reasonably small number of holes are considered, wall-resolving LES can be performed in place of RANS calculations mainly to gain insights into the jet/mainstream interaction. Only a few LES studies have been performed for multiple rows with geometrically resolved 30 jet/jet interactions. Motheau et. al. 12] carried out a LES study on a multi-row cooling configuration. Their main focus was on the interaction of an acoustic wave with the coolant jets rather than the cooling effectiveness. Konopka et al. [13] analysed the interaction of multiple rows of coolant jets using LES. The flow field comparisons against the Particle Image Velocimetry (PIV) data showed good agreement but no assessment was made for the cooling effectiveness. However, because of the requirement of LES 35 in near-wall grid resolution, $N \propto R e^{2.4}$, wall-resolving LES studies are limited to low Reynolds number configurations.

Scaling the combustor conditions can be very useful. In recent years, experimental studies are carried out by Martin [14] and Krawciw et. al. [15, 16] assessing the performance of full coverage effusion cooling array configurations at scaled combustor conditions. In the former work, a jet-in-crossflow type turbulence generator was built to generate combustor relevant flow conditions with high intensity levels and large lengthscales. The cooling performance of cylindrical and fan-shape effusion cooling holes was studied by adiabatic cooling effectiveness (ACE), normalised heat transfer coefficient (HTC) and heat flux reduction (HFR). It was found that the free-stream turbulence levels can increase the rate of mixing and degrade the cooling effectiveness at low blowing ratios $(B R)$. At high $B R \mathrm{~s}$, the surface ACE was 45 increased due to the reattachment of the coolant jet caused by the mainstream turbulence. It was also found that the effect of density ratio $(D R)$ as an independent parameter was relatively weak in inclined cylindrical hole cases. The ACE results of $D R$ from 1 to 1.4 scale reasonably well against momentum flux ratio, which suggests that the results obtained at low DR can be used to predict that at combustor $D R$. The fan-shape hole was found to improve the spatially averaged ACE by $89 \%$. This is mainly due to the diffusion of the coolant at hole exit which keeps the coolant jet attached to the plate surface at $B R$ s up to 5. Krawciw [15, 16] extended the work further to different hole geometries ranging from the most simple inclined cylindrical hole to more exotic helical hole. The cooling performance of those 
designs were examined by both the ACE in [15, 16] using a Pressure-Sensitive-Pain (PSP) technique and the overall cooling effectiveness (OCE) accounting the heat conduction inside the effusion-cooled plate in [16] using the Infra-Red Thermography. The experiments in [15] were conducted at momentum ratios $(M R)$ ranging from 0.6 to 15 . It was found that the fanned-shape related designs generally performed better than the cylindrical holes in terms of ACE and coolant film coverage. Also, the averaged ACE results vary little when $B R$ exceeds 1.5 for most of the designs.

In this paper, a wall-proximity blended hybrid LES-RANS approach is used to conduct numerical simulations with one of the multi-row effusion cooling configurations in Krawciw's experiments [15]. The hybrid LES-RANS approach is used to reduce the computational expenses in the near-wall region by the use of RANS as near-wall models, but resolving the turbulent flow features that are not attached to the wall 17 19]. The main purpose of this study lies in two folds. Firstly, the numerical approaches are examined by the prediction of the surface ACE distribution. Secondly, investigations are carried out based on the eddy resolving solutions to study the flow behaviour and the formation of the coolant film in this multi-row configuration at scaled combustor conditions. Additionally, the effects of turbulent flow structures are also examined.

The paper is organised as follows. The methodology and configuration for the simulations are explained in Section 2, including explanation of the grid resolution and summary of the cases. Section 3 describes the time-averaged flow field obtained by the simulations. Results of the surface ACE distribution compared with the measurements and RANS solutions are presented in Section 4. Detailed study of the coolant film formation and coolant jet development is also presented in this section. Section 5 illustrates analysis on the turbulent flow structures in the coolant-mainstream mixing region and off-wall region, as well as brief discussion about their effects on the surface ACE distribution and coolant film formation.

\section{Methodology and configuration}

\subsection{The unstructured in-house solver}

The current study employs an in-house hybrid LES-RANS solver for unstructured grid, FLUXp (Flux Limited Unstructured eXtrapolation in parallel) [20, 21]. In this solver, the Favre-averaged compressible

Navier-Stokes equations for ideal gas are solved in conservation form,

$$
\frac{\partial}{\partial t} \int_{\Omega} \mathbf{W} d V+\oint_{\partial \Omega}(\mathbf{F}-\mathbf{G}) \cdot \mathbf{n} d A=0
$$

The conservative variables are $\mathbf{W}=\left[\rho, \rho u_{i}, E\right]^{T}$, the inviscid and viscous fluxes are defined as $\mathbf{F}_{i}=$ $u_{i} \mathbf{W}+\left[0, \delta_{1 i} p, \delta_{2 i} p, \delta_{3 i} p, u_{i} p\right]^{T}$ and $\mathbf{G}_{i}=\left[0, \tau_{1 i}, \tau_{2 i}, \tau_{3 i}, \tau_{k i} u_{k}+q_{i}\right]^{T}$. The stress tensor $\tau_{i j}$, total energy $E$ and heat flux $q_{j}$ satisfies,

$$
\tau_{i j}=2\left(\mu+\mu_{T}\right)\left(S_{i j}-\frac{1}{3} \partial_{k} u_{k} \delta_{i j}\right), \quad E=\rho e+\frac{1}{2} \rho u_{i} u_{i}, \quad q_{i}=-\left(\kappa+\kappa_{T}\right) \frac{\partial T}{\partial x_{i}}
$$

where the thermal conductivity is computed from $\kappa=\mu c_{p} / \operatorname{Pr}$. The Prandtl number is set to $\operatorname{Pr}=0.72$ and its turbulent counterpart is 0.90. An extra equation of state $p=\rho R T$ is also included. In order to overcome the low Mach numerical stiffness, a preconditioned formula is more robust and more details of the preconditioning is presented in Appendix A. 


\subsubsection{Spatial and temporal discretisation}

Viscous terms are discretised using the central differencing scheme, while a MUSCL type reconstruction with a modified Roes scheme is used for inviscid fluxes at the common face of two neighbouring cells,

$$
\mathbf{F}=\frac{1}{2}\left(\mathbf{F}_{R}+\mathbf{F}_{L}\right)-\frac{\gamma}{2}\left|\frac{\partial \mathbf{F}}{\partial \mathbf{Q}}\right|\left(\mathbf{Q}_{R}-\mathbf{Q}_{L}\right)
$$

where the up-winding term is controlled by parameter $\gamma \in(0,1]$. When $\gamma=0$, the central difference is used. Meanwhile, the upwinding is fully functioning when $\gamma=1$. The blending between the two schemes is applied to combine the accuracy of the central difference scheme in resolving the turbulent flow structures, and the numerical stability of the upwinding scheme. In the current simulations, $\gamma \approx 0.3$ is defined in regions covering the coolant jets and their wakes, and is smoothly transitioned to 1 in the farfield. $\mathbf{Q}=\left[p, u_{i}, T\right]^{T}$ and $\mathbf{F}$ are the primitive and flux vectors. Subscripts " $L$ " and " $R$ " represent the immediate left and right position of the common flux face, where piecewise linear reconstructions are performed from cell centres to give a second-order spatial accuracy. Moreover, the dual-time advancing is employed with the outer physical time discretised by a three-level backward Euler scheme, thus leading to a second-order temporal accuracy. The inner pseudo time is advanced by a three-stage Runge-Kutta scheme. As the outer time is formulated implicitly, it allows larger physical time steps to increase the computing efficiency compared to its explicit counterpart.

\subsubsection{LES-RANS hybridisation}

In this work, the RANS model is used in the near-wall region instead of a wall-resolving LES approach to reduce the requirement of near-wall grid resolution. A wall proximity formula is used to control the "destruction" of the eddy viscosity in the RANS part [22]. This is different from the Detached Eddy Simulation (DES), where the approximate turbulent lengthscale is replaced by the weighting between the lengthscale and grid spacing $\min \left(L_{T}, C_{D E S} \Delta\right)$ [23 25]. In DES, the interface between the LES and RANS is not fixed but depends on the flow and grid resolution. However, in our hybrid LES-RANS approach, the LES-RANS interface is determined at a given wall proximity based on a RANS cut-off distance $d_{c}$, or the corresponding $y^{+}$value. And the LES and RANS regions are defined by a modified wall distance,

$$
\widetilde{d}=\left[1-\tanh \left(\alpha \frac{d-d_{c}}{d_{c}}\right)\right] \frac{d}{2}
$$

where $\alpha$ is a coefficient which controls the decay rate of $\widetilde{d}$ in the mixed region, $d_{c}$ is the RANS cut-off

distance typically corresponding to $y^{+} \sim 60$. The weighting parameter for LES is given as,

$$
\varepsilon=\tanh [\beta(d-\widetilde{d})]
$$

where $\beta$ is a coefficient that controls the growth of $\varepsilon$ in the mixed region, increasing the value of $\beta$ will result in a faster increase of $\varepsilon$ before reaching the maximum value of 1 . The LES weighting parameter is used when calculating the SGS eddy viscosity,

$$
\mu_{s g s}=\varepsilon \mu_{s m a g}
$$


where $\mu_{s m a g}=\rho l_{s g s} D_{s}$ is the eddy viscosity computed from the Smagorinsky-Lilly model [26], where

$l_{s g s}=C_{s} \Delta$ is the sub-grid scale, and $D_{s}=|\bar{S}|$ is model differential operator. This eddy viscosity can also be replaced by the eddy viscosity from other SGS models, for example $\mu_{\sigma}=\rho l_{s g s} D_{\sigma}$ from the $\sigma$-model 27].

The two-equation Shear Stress Transport (SST) $k-\omega$ model [25] is used as the near-wall RANS model. The transport equations of the SST model and definitions of the variables are presented in Appendix B with details. In order to blend the SST model with the LES, the turbulent length scale $L_{T}$ in the "destruction" term $B_{k}$ of the transport equations is modified so that the eddy viscosity of the RANS model $\mu_{T}$ is controlled by the wall proximity $\widetilde{d}$,

$$
B_{k}=k^{3 / 2} \frac{1}{L_{T}} \frac{d}{\widetilde{d}}
$$

The turbulent length scale $L_{T}$ therefore scales with the modified wall distance in the mixed region but returns to the original value in the RANS region.

As Figure 1 shows, the RANS and LES regions are defined based on the modified wall distance $\widetilde{d}$. The eddy viscosity of RANS reduces smoothly when moving away from the wall in the mixed region. The weighting of LES increases in the mixed region when $\varepsilon$ gradually increases from 0 to 1 . In the LES region where $\varepsilon=1$, the RANS eddy viscosity vanishes, and the SGS eddy viscosity has full weighting. A smooth transition in the eddy viscosity field is therefore obtained between the RANS and LES regions, and the effective viscosity being used during the simulation is calculated,

$$
\mu_{e f f}=\mu_{\text {lam }}+\mu_{T}+\mu_{\text {sgs }}
$$

where $\mu_{\text {eff }}$ is the effective viscosity, $\mu_{\text {lam }}$ is the dynamic (laminar) viscosity. Also, it has been shown that the near-wall RANS has maintained its ability to reproduce the log-law near-wall behaviour [20, 22, 28].

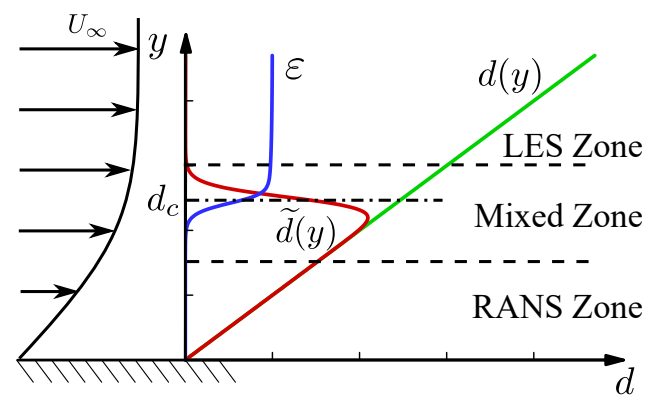

Figure 1: Blending of RANS and LES regions

In this work, since no explicit SGS model is used, $\mu_{s g s}$ is zero, which is behaving like an implicit LES 29 31] or numerical LES 32]. This hybrid LES-RANS approach has been successfully applied to various studies, such as turbulent jets [18, 21] and a heated bluff body flow [19].

\subsection{Krawciw's experiments}

Experiments were carried out by Krawciw et al. [15, 16] on the "Biot-Scale Rig" designed by Martin 14] at Loughborough University. This rig was developed to run Biot number matched experiments, 
replicating the overall effectiveness of combustor representative cooling configurations. According to

15], the combustor conditions are aero-thermally scaled to the near-ambient rig conditions due to the limit of the wind tunnel. In order to represent the exact combustor equivalent conditions, a so-called "Biot-number-scaling" is applied when determining the test conditions. Firstly, the Reynolds number of the experiment must match that of the combustor conditions to ensure that the flow field is similar. Secondly, Nusselt number must be matched to scale the boundary between the fluid and the wall, as well as the Biot number, to reproduce the conduction of heat inside the plate, for furture studies on the overall cooling effectiveness. Therefore, the fluid properties, convective heat transfer and plate conductivity must be scaled to match the Reynolds number, Nusselt number and Biot number of the combustor conditions. Moreover, the facilities were modified by Krawciw et al. [15] to expand the capability and fidelity. A few additional equipment were added, including a bulk free-stream turbulence generator and those for pressure sensitive pain (PSP) measurement. A range of geometric scales and measurement of ACE were available after the modification. The mainstream air in the wind tunnel was provided through a recirculating wind tunnel with an in-line heater. The mainstream entered a turbulence generator after a series of flow straightening grids and screens. The turbulence generator took air from the downstream of the inline heater and re-entered the mainstream through a set of holes with variable diameters. By varying the jet velocity and hole diameter, different bulk turbulence intensity levels in the mainstream could be generated. Details of the jet-in-crossflow type turbulence generator can be found in [14]. The coolant flow was fed into a plenum before entering the coolant holes from a separate source. The speed of the coolant flow was controlled by flow controllers and the plenum was made of materials with limited conduction rate. Downstream the test section, the wind tunnel was vented to atmosphere in order to maintain the atmospheric pressure and prevent heat build-up and Nitrogen concentration during the experiment.

\subsection{Flow domain and boundary conditions}

A sketch of the computational domain is illustrated in Figure 2a It contains two columns of the coolant holes with a spanwise distance of 3.1D. Using the periodic boundary conditions at the two sides of the mainstream channel, this section can be extended periodically in the spanwise direction, mimicking the full plate in experiments. The top surface of the main channel locates about $7.75 D$ away from the plate surface and it is defined as far-field to allow flow penetration through the boundary. The origin of the computational domain is set at the centre of the two rows and parallel to the leading edge of the first coolant hole. The inlet locates at about 11.6D away from the leading edge of the first hole and the outlet is about $15 \mathrm{D}$ away from the trailing edge of the last hole. The bottom surface of the main channel, together with the coolant hole surface and top surface of the coolant chamber, is set as no-slip and zero heat flux wall. This ensures that no extra heat is introduced to the domain and that the wall surface is adiabatic. The coolant holes stretch $3.1 D$ in the vertical direction with an very shallow inclination angle of around $17^{\circ}$ to the plate surface, which leads to a length-to-diameter ratio of about 10.6D. The coolant enters the chamber from the bottom surface that is about $4.65 \mathrm{D}$ away from the coolant hole inlet. The surfaces of 4 sides are slip walls to make sure that all the coolant that enters the chamber will come out 
from the coolant holes.

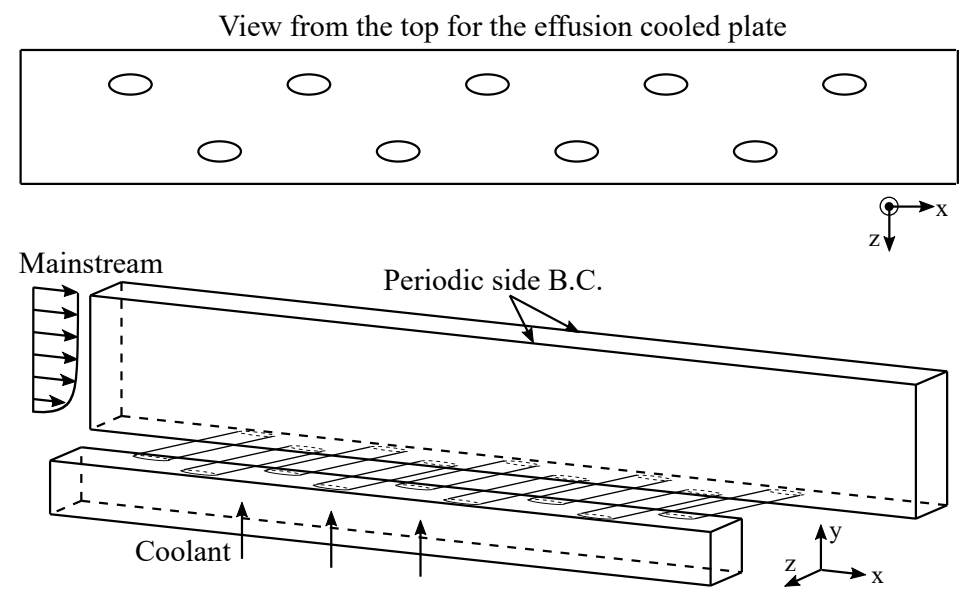

(a) A sketch of the computational domain and boundaries

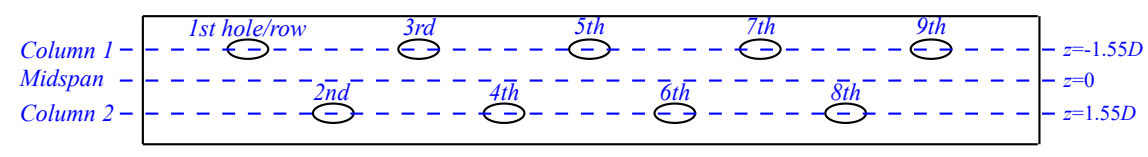

(b) Definition of the coolant hole locations

Figure 2: A sketch of the computational domain and the effusion-cooled plate

According to the experiment [15], the mainstream bulk velocity is $U_{\infty}=36 \mathrm{~m} / \mathrm{s}$ with a velocity profile specified at the inlet. The coolant speed at the inlet of the chamber is adjusted to reach the targeted blowing ratio at the coolant hole exit. The mainstream temperature is $T_{\infty}=315 \mathrm{~K}$ while the coolant flow has a temperature of about $T_{c}=300 \mathrm{~K}$. This leads to a density ratio of 1.05 and a blowing ratio of around $B R=1.5$, with corresponding Reynolds number of around $R e_{D} \approx 6,000$, which are equivalent to the experiments. A velocity profile is used at the inlet of the mainstream, with synthetically generated free-stream turbulence using the Synthetic Eddy Method (SEM) 33 35] at a turbulent intensity level of $5 \%$, and a lengthscale of about $2 D$.

\subsection{Mesh and case summary}

Two coarse grids of about 3-million and 8-million cells are used for RANS simulations. The coarse 3-million-cell grid do not have specific refinement region for the turbulent flow structures. A maximum cell size of $1.2 D$ is applied to the farfield of the main flow domain, while that on the effusion cooled plate surface is limited to $0.4 D$. The surface cells are growing to the maximum surface cell size in this 3-million-cell grid without any specific restriction. Prism layers are generated from the solid surfaces with the first cell height set to satisfy $y^{+} \approx 1.0$ based on the mainstream inlet conditions. Additionally, a cell-to-cell match condition is applied to the left and right boundaries in the spanwise direction of the mainstream channel to comply with the requirement of the periodic boundary condition. In the 8-millioncell grid, refinement regions are used to limit the volume cells size to a maximum of $0.20 \mathrm{D}$ in the regions covering the injected coolant jets and their wake where the coolant jets mix with the mainstream. This refinement aims at better resolving the shear layer and the mixing of coolant jets and the mainstream, 
which are important to the formation of the coolant film. Cells on the plate surface and in the near-wall regions are refined to capture the injection and reattachment of coolant jets. The resolution of those highly unsteady flow structures are important to the prediction of surface ACE distribution. In this 8-million-cell grid, the cell size on the coolant hole exit edges is the same as the that of the 3-million-cell grid, but the growth of the cell size in the middle regions between the coolant holes is controlled by a maximum size of $0.18 D$, similar to the cell size in the volume refinement regions. A much finer grid of about 18-million cells is used for the hybrid LES-RANS case. The refinement regions are the same as the 8-million-cell grid, but the resolution is much finer. The cell size in the volume refinement regions is set to $1 / 5$ of the value used in the 8-million-cell grid, which is about $0.04 D$. Meanwhile, the cells on the plate surface between the coolant holes are limited to about $0.08 D$, which is about half of the corresponding value in the 8-million-cell grid. These refinement processes result in a volume resolution in the corresponding regions to be more than doubled compared to the 8-million-cell grid. Figure 3 shows an overall view and an enlarged cut-away view of the 18-million-cell grid for eddy resolving simulation.

A summary of the simulations carried out in the current study is presented in Table 1, in which $N_{\text {cell }}$ is the total cell number, $\Delta_{V}$ and $\Delta_{S}$ are the maximum cell size in the volume and plate surface refinement regions, $\Delta t$ the time step size and the convective time is defined as $t^{*}=D / U_{\infty}$. The timestep size in MRC3 is set to be $1 / 200$ of the convective time. To evaluate the timestep size being used in the simulation, an maximum equivalent CFL number is calculated based on the bulk velocities and the minimum cell size, although the implicit method being used in the solver allows much larger CFL number compare to the explicit method $\left(C F L_{\max } \leqslant 1\right)$. In MRC3, the maximum equivalent $\mathrm{CFL}$ number with the mainstream velocity is about $C F L_{U_{\infty}} \approx 0.1$, and that with the coolant jet velocity is about $C F L_{U_{c}} \approx 0.6$.

Table 1: A summary of simulations performed in the current study

\begin{tabular}{ccrccc}
\hline \hline Case & Approach & $N_{\text {cell }}$ & $\Delta_{V}$ & $\Delta_{S}$ & $t^{*} / \Delta t$ \\
\hline MRC1 & RANS & $2,830,211$ & $1.20 D$ & $0.40 D$ & N/A \\
MRC2 & RANS & $7,661,401$ & $0.20 D$ & $0.18 D$ & N/A \\
MRC3 & hybrid LES-RANS & $17,866,961$ & $0.04 D$ & $0.08 D$ & 200 \\
\hline \hline
\end{tabular}

\section{Time-averaged flow field}

Detailed study of the time-averaged flow field can be carried out based on results of the simulations both for the temperature and velocity field.

\subsection{Examination of the temperature field}

The time-averaged temperature field is studied first for a brief overview of the mixed flow field formed by the coolant jets and the mainstream. Fig. 4 illustrates the mean temperature contours at a slice through the first column of holes $(z=-1.55 D)$. The time-averaged temperature fields across the 1st column of coolant holes from the 3 cases are very similar to each other. The thickness of the coolant 


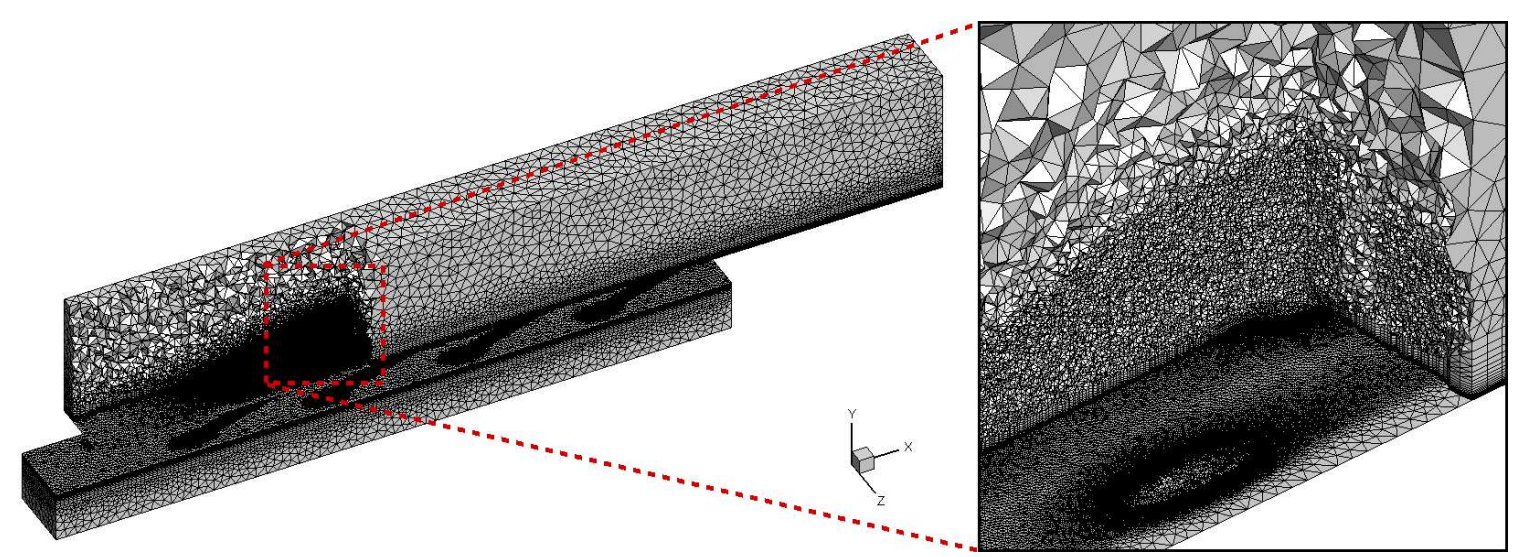

Figure 3: The MRC3 grid: a general view of the cut-away position (left) and an enlarged view showing detailed grid resolution (right)

film, denoted by the position of the upper shear layer, is growing smoothly from the upstream coolant hole to the downstream ones. It can be found from all 3 cases that the coolant jet from the first hole does not reattach to the plate surface very well. This results in a high flow temperature region occurring near the plate surface downstream the first hole's exit. This is due to the coolant jet penetrating deeply into the mainstream as a result of the high momentum ratio of the current configuration, in spite of the small jet inclination angle. Some differences in the near-wall region can be found between the 3 cases. Firstly, the lower shear layer of the 1st coolant jet in MRC1 goes straight up in the streamwise direction, while that in the other two cases shows a trend of reattachment. This suggests that MRC1 does not have enough resolution in the region close to the wall to capture the reattachment of the coolant jet, thus underpredicts the off-wall flow mixing as well as the surface ACE value. MRC2 presents a trend of reattachment, but the strength of the reattachment is not as well predicted as in the eddy-resolving

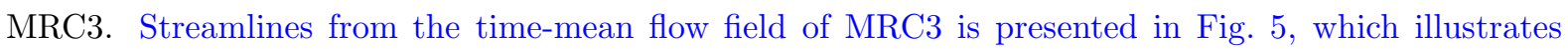
the separation and reattachment of the coolant flow. Secondly, the decreasing speed of the near-wall flow temperature towards the downstream direction is underpredicted in MRC1 and MRC2, due to the underestimate in the coolant jet reattachment. Case MRC3 shows a clear decreasing trend in the nearwall flow temperature. The coolant flow fully reattaches to the plate surface after the third hole. It seems that the downstream coolant jets are more attached to the plate surface. This is highly likely due to the effects of a high-velocity and low-temperature coolant film formed by the wake of the upstream coolant jets. Furthermore, the comparison between the downstream coolant jets to the upstream ones indicates that the low temperature core of the downstream ones extends further in the streamwise direction. This is also because of the effects of the low-temperature coolant film attached to the plate surface. The heat transfer rate between the coolant film and the coolant jets is reduced since the temperature difference is reducing as more coolant is injected into this coolant film. As a result, the low temperature core becomes increasingly longer in the downstream coolant jets.

Fig. 6 presents the mean temperature contours on the plate surface and selected downstream slices in MRC3. It can be found that the coolant jets of the first 2 rows are lifted off the plate surface, thus would result in a high temperature flow attached to the surface locally. Therefore, a low value of the 


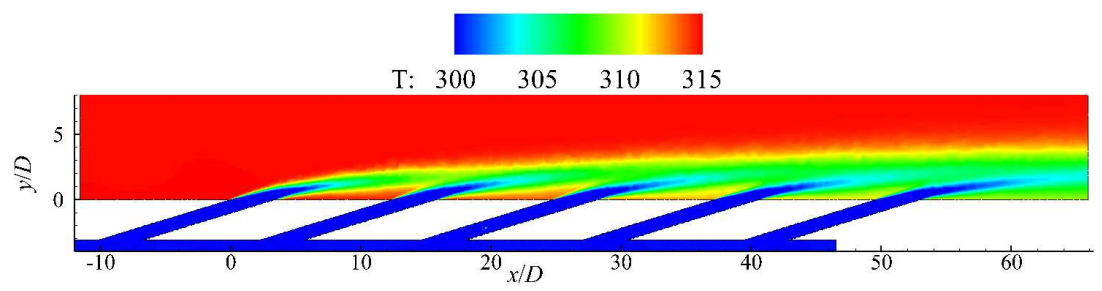

(a) MRC1

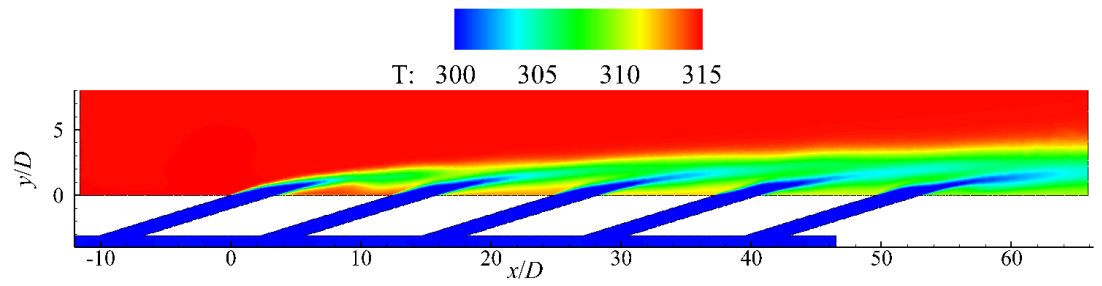

(b) $\mathrm{MRC} 2$

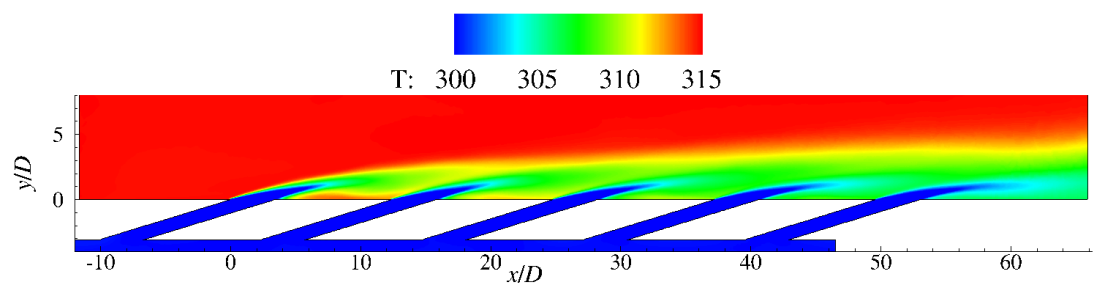

(c) MRC3

Figure 4: Time-averaged temperature contours at a slice through the first column holes of: (a) MRC1, (b) MRC2, (c) MRC3

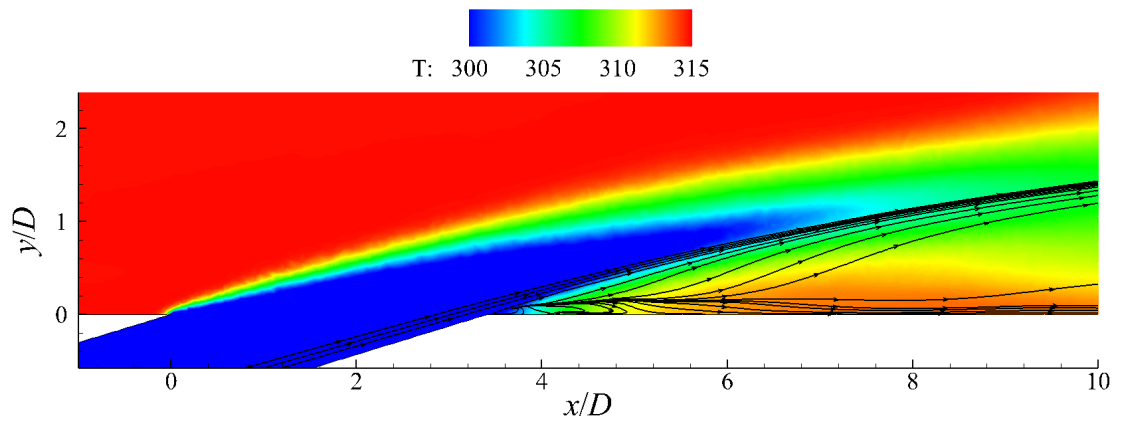

Figure 5: Streamlines of the separated and reattached coolant from the 1st coolant jet in MRC3

surface ACE is expected in the position corresponding to the first two rows. After the 3rd row, the coolant jets are pressed onto the plate surface by the coolant film which is a mixture of the upstream coolant jets and part of the mainstream. As a result, the flow temperature attached to the surface is lower since more coolant is reattached to the surface. Moreover, the overall temperature of the coolant film is reduced as more coolant is injected to the mainstream, thus compensates the heat transferred to the coolant film from the hot mainstream. It is also noticeable that the spanwise coverage of the coolant film keeps increasing since the 4th row. The most possible reason of this expansion is that the local coolant jet is forced to expand further and further in the spanwise direction in response to the constrain 
of the incoming coolant film in the vertical direction.

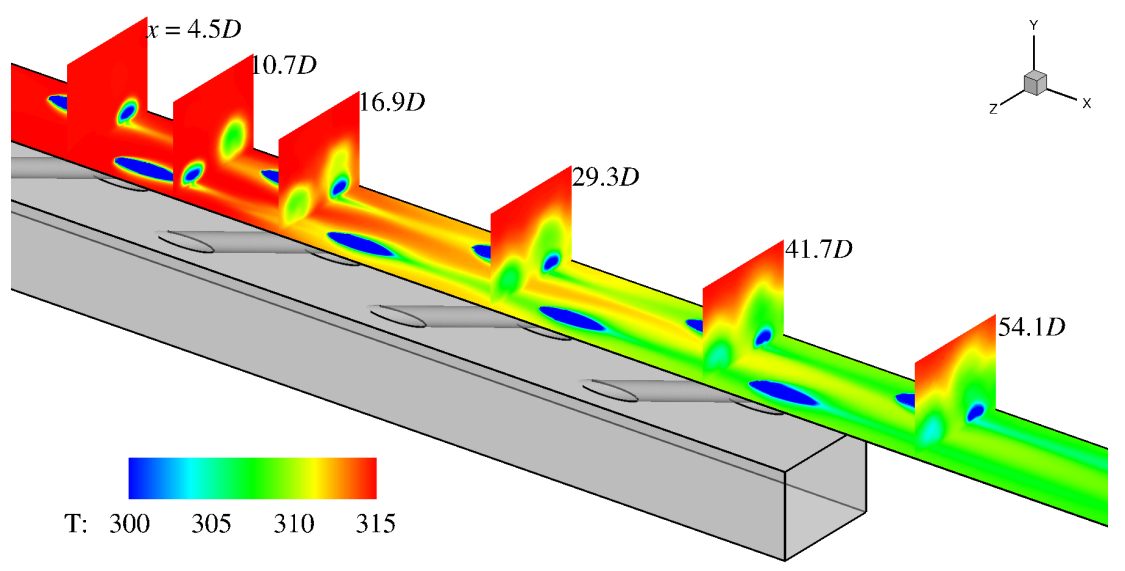

Figure 6: Time-averaged temperature contours on the plate surface and selected downstream slices of MRC3

\subsection{Examination of the velocity field}

The mean flow fields of the simulations are examined by the velocity and Reynolds stresses profiles. The velocity field across and in the wake of the first coolant hole is studied to provide a basic understanding of the mixing flow between the coolant jet and the mainstream at current conditions. The development of the flow field with multiple coolant jets is illustrated by the velocity and Reynolds stress profiles in the wake of each coolant hole through the centre of the first column at $z=-1.55 D$. It is worth noting that the Reynolds stresses from MRC3 are resolved in the LES regions, while those from MRC1 and MRC2 are modelled by RANS.

Figs. 7 and 8 present the mean streamwise and vertical velocities through the centreline of the first coolant hole. The origin of the streamwise position is at the leading edge of the first coolant hole and the trailing edge of the first coolant hole is at $x=3.42 D$ as illustrated in the figure. The mainstream boundary layer is clearly illustrated by the streamwise velocity profile at the leading edge of the coolant hole $(x=0)$. It shows that in MRC1 and MRC2, the bulk velocity in the farfield is slightly underpredicted. At $x=2 D$, which is slightly downstream the centre of the coolant hole exit, the coolant jet is exiting the hole exit at a streamwise velocity of about $2 U_{\infty}$ and a vertical velocity of about $0.5 U_{\infty}$. The vertical velocity is slightly lower in MRC1 due to the coarse resolution. Similar underestimate is also found in $\mathrm{MRC} 2$, but is able to recover in a higher position. An upper shear layer is formed at about $y=0.5 \mathrm{D}$ locally. This shear layer position moves up in the downstream as the coolant jet penetrates further into the mainstream. The coolant jet core is illustrated by the wide peak in the velocity profiles downstream the trailing edge. However, MRC1 and $\mathrm{MRC} 2$ underpredict the jet core velocity correspondingly. A recirculation region is formed in the near-wall region close to the trailing edge of the coolant hole, as the negative vertical velocity in the profiles at $x=4 D$ and $6 D$ indicate. This recirculation region is generated by the separation of the coolant jet from the trailing edge of the coolant hole. It ends after $x=6 D$ as the near-wall vertical velocity at $x=8 D$ is no-longer negative, which indicates that the separated coolant flow fully reattaches to the plate surface. 


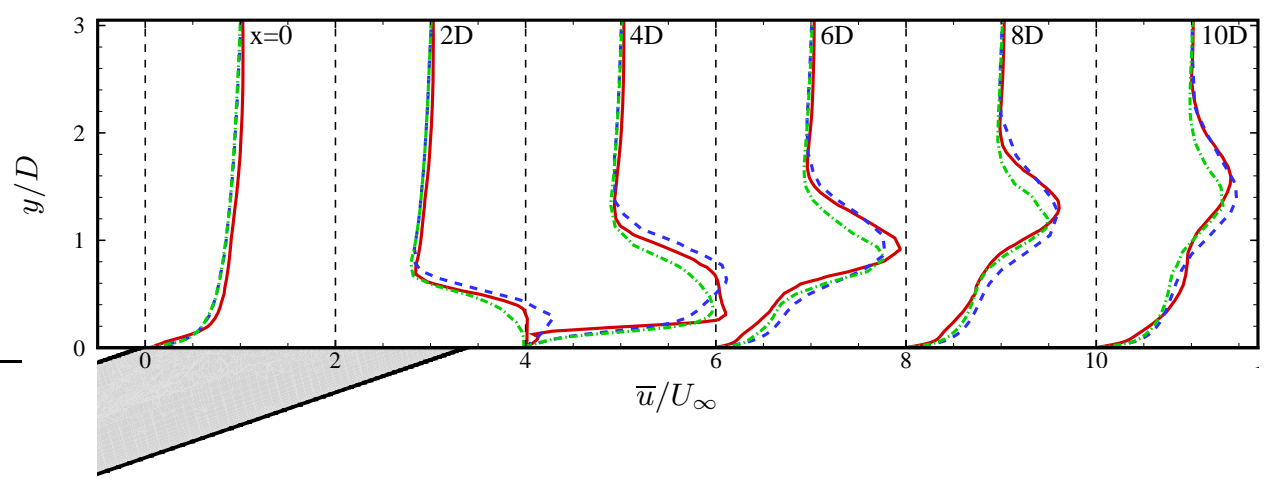

Figure 7: Time-averaged streamwise velocity profiles at $z / D=-1.55$ through the 1 st coolant hole, $-\cdot-$ MRC1, -MRC2, — MRC3

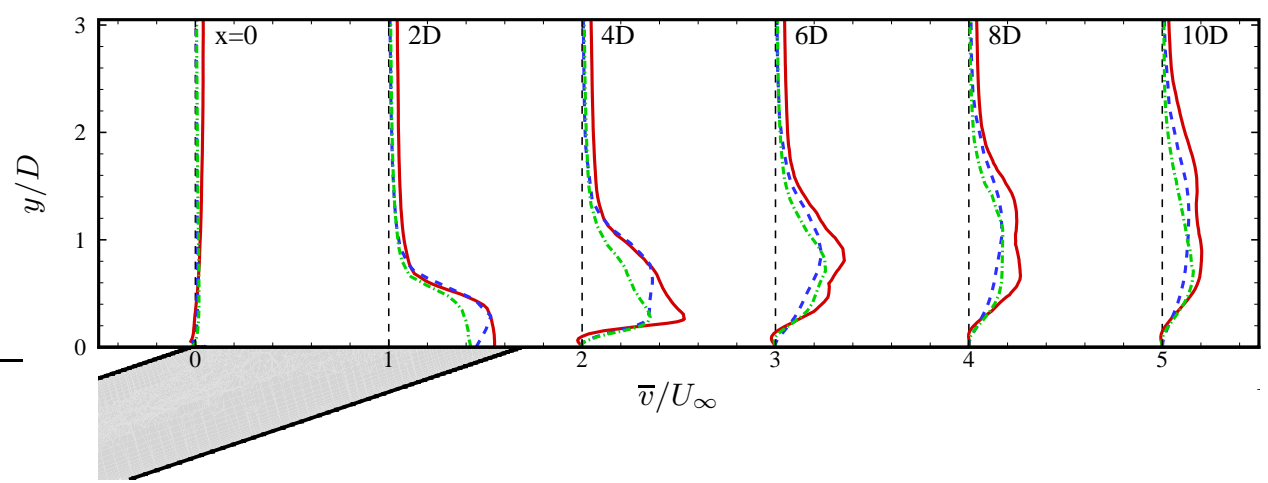

Figure 8: Time-averaged vertical velocity profiles at $z / D=-1.55$ through the 1st coolant hole, legend same as Fig. [7]

Profiles of the Reynolds normal stress $\left(\overline{u^{\prime} u^{\prime}}\right)^{1 / 2}$ of the first coolant hole are illustrated in Fig. 9] The profiles indicate that turbulence intensity of $5 \%$ added to the mainstream is present in all three cases across the 1st coolant hole. Comparison between profiles of the three cases suggests that MRC1 and MRC2 completely underestimate the turbulent flow features, especially in the upper and lower shear layers. Though the grid refinement in MRC2 help providing better prediction to the turbulence, the improvement is limited and cannot compare to the eddy resolving MRC3. A clear peak can be found in the profile of $x=2 D$ at about $y=0.5 D$. This indicates that the velocity at the upper shear layer is fluctuating significantly. The peak moves up towards the downstream, but the transition is smoother

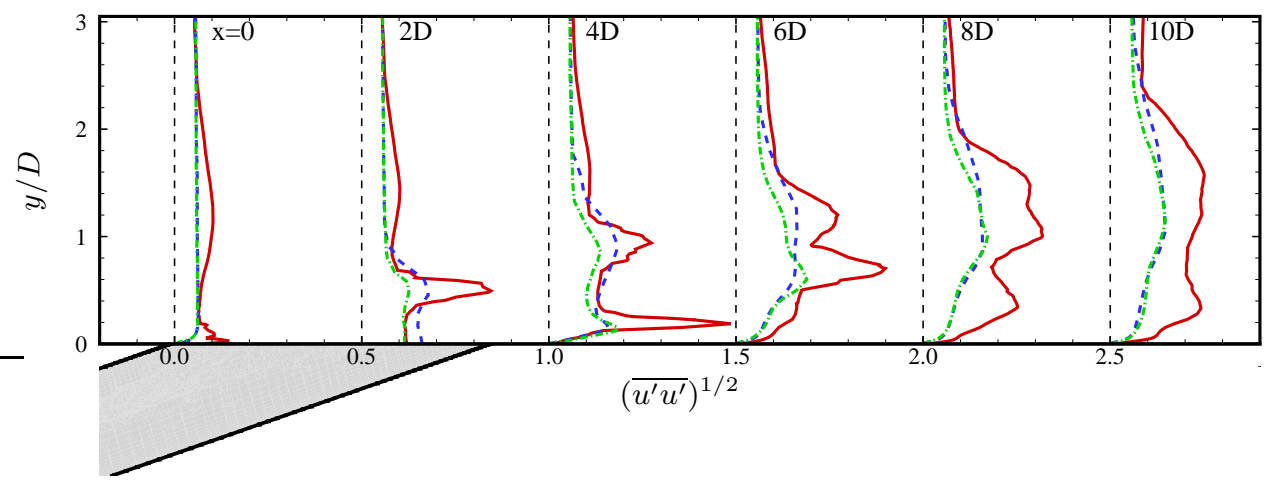

Figure 9: Reynolds normal stress $\left(\overline{u^{\prime} u^{\prime}}\right)^{1 / 2}$ profiles at $z / D=-1.55$ through the 1 st coolant hole, legend same as Fig. 7 
due to the shear layer breaking up into smaller structures. A second peak appears close to the wall at $x=4 D$. This is due to the formation of another shear layer between the lower boundary of the coolant jet and the mainstream. Similar to the upper shear layer, its position moves up and the strength reduces during the breakup. A third but less strong peak occurs at $x=8 D$. This peak is probably caused by the unsteadiness of the separated flow from the coolant jet.

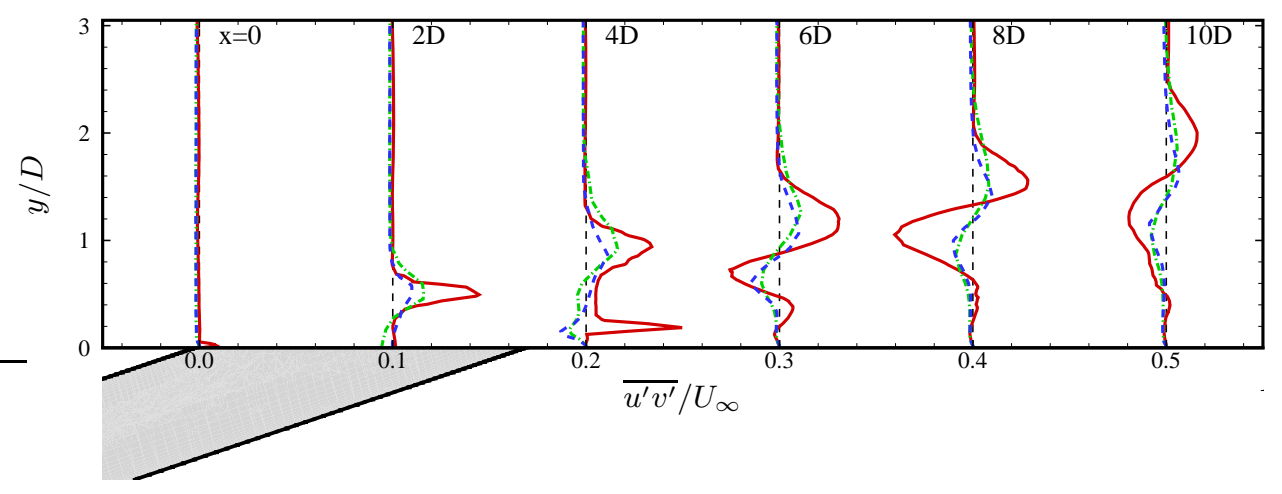

Figure 10: Reynolds shear stress $\overline{u^{\prime} v^{\prime}}$ profiles at $z / D=-1.55$ through the 1 st coolant hole, legend same as Fig. 7

Fig. 10 shows the Reynolds shear stress $\overline{u^{\prime} v^{\prime}}$ across the first coolant hole and at downstream locations. Similar to the profiles of the Reynolds normal stress $\left(\overline{u^{\prime} u^{\prime}}\right)^{1 / 2}$, instability in the upper and lower shear layers of MRC1 and MRC2 is not well resolved compared to that in MRC3. The upper and lower shear layers are illustrated by the positive peaks at $x=2 D$ and $4 D$. In the downstream regions, the lower shear layer is demonstrated by a negative peak. The change in the peak direction of the lower shear layer between $x=4 D$ and $8 D$ is due to the fact that the wall boundary layer formed inside the coolant hole still exists at $x=4 D$, but is smoothed out at $8 D$. This wall boundary layer introduces a different streamwise velocity distribution compared to that of the standard shear layer. The movement of the upper shear layer in the vertical direction is illustrated much clearer in the shear stress profiles. The upper shear layer moves up to about $y=2 D$ at downstream location of $x=10 D$. This suggests that at such a high momentum ratio, the coolant jet penetrates deeply in vertical direction, in spite of the small hole inclination angle of $17^{\circ}$. The upper boundary of the coolant jet is so far from the plate surface that only a small amount of coolant is attached to the wall. This is one of the reasons why the surface ACE remains low in the wake of the first 3 holes.

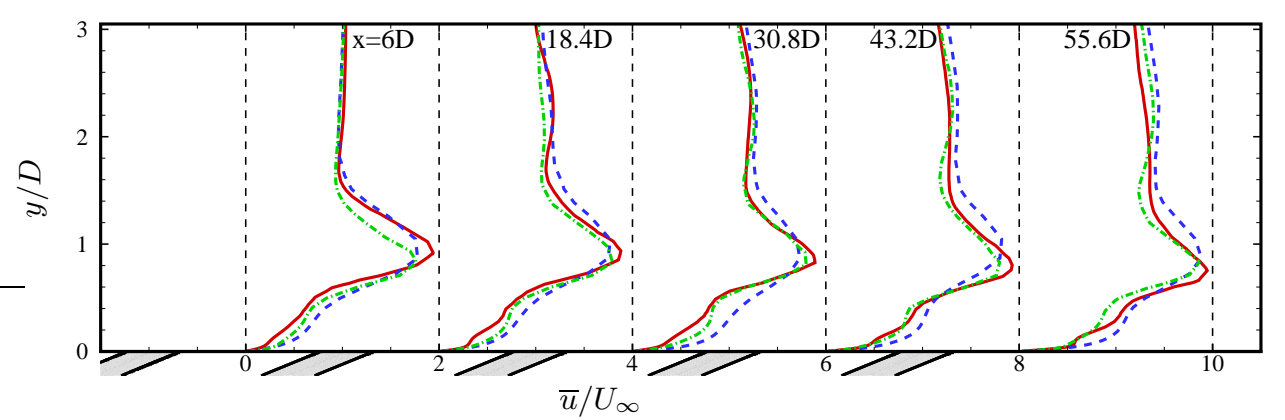

Figure 11: Time-averaged streamwise velocity profiles at $z / D=-1.55$ through the first column of holes, legend same as Fig. 7 
The mean streamwise velocity in the wake of the 5 coolant holes in the first column is illustrated in Fig. 11. The peak values of the coolant jet cores are again underpredicted in MRC1 and MRC2, though the reducing trend of the peak velocity and its vertical position being captured. The coolant jet core, demonstrated by the wide peak in the velocity profile, is at around $y=1 D$ in the wake of the first increase in the local velocities results in a decrease on the blowing ratio of the local coolant jet. This is one of the reasons why the downstream coolant jet become more attached to the plate surface.

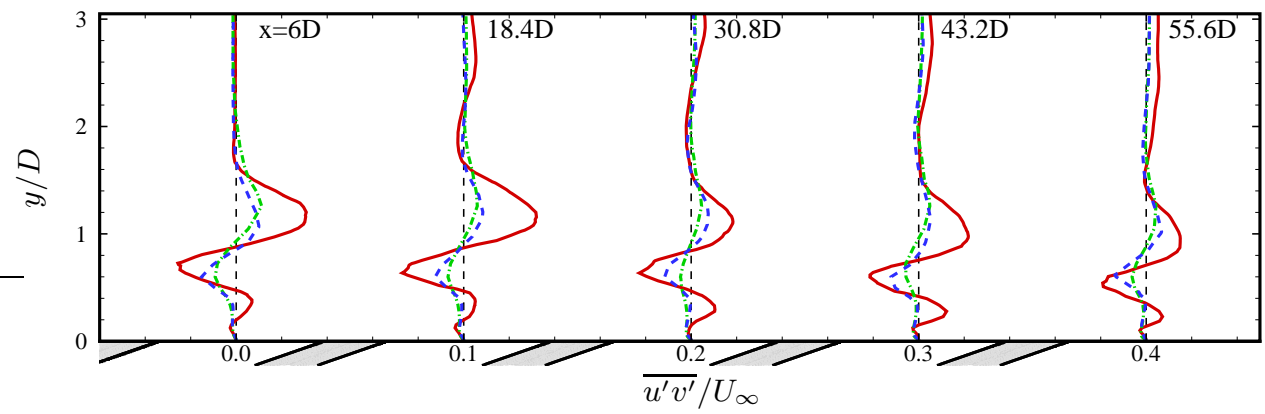

Figure 12: Reynolds shear stress $\overline{u^{\prime} v^{\prime}}$ profiles at $z / D=-1.55$ through the first column of holes, legend same as Fig. 7

Fig. 12 shows the Reynolds shear stress $\overline{u^{\prime} v^{\prime}}$ in the wake of the 5 coolant holes in the 1 st column. The upper and lower boundary of the coolant jet core, or the upper shear layer of the coolant jet, is illustrated by the first pair of peak and valley at about $y=1 D$. The value of the peak and valley reduces towards the downstream as a result of the increasing velocities in the coolant film consists of multiple coolant jets. Although its presence and decaying trend is also shown in MRC1 and MRC2, the strength of the instability is greatly underpredicted. The second pair of peak and valley, although not as high as the first pair, indicates the lower boundary of the coolant flow, or the lower coolant jet shear layer, where some of the coolant flow separates from the main jet and reattaches to the wall. As a result of the increasing velocity in coolant film towards downstream, both the coolant jet core and the separated coolant flow region become more and more closer to the wall, with a reduction in the unsteadiness. Comparing to MRC3, MRC1 and MRC2 failed to capture such flow separation and reattachment very close to the plate surface, which would greatly affect the results of surface ACE distribution.

\section{Coolant film distribution}

In this section, the distribution of the coolant film over the plate with multiple rows of coolant holes is studied using the time-averaged adiabatic cooling effectiveness. And the hybrid LES-RANS results are compared with RANS solutions and relevant measurements. More detailed investigation on the coolant 
film formation throughout the multiple rows and its three dimensional structure are also investigated by a coolant film thickness defined later in this section. Differences on the coolant jet injection between the upstream and downstream holes are also studied with the help of the coolant jet mean centreline trajectory.

\subsection{Adiabatic cooling effectiveness}

Contours of the mean surface ACE of the simulations and the corresponding measurements are shown in Fig. 13. The surface ACE is calculated using the mainstream temperature $T_{\infty}$, coolant temperature $T_{c}$ and local wall temperature $T_{w}$,

$$
\eta=\frac{T_{\infty}-T_{w}}{T_{\infty}-T_{c}}
$$

Comparing the results of three cases, it is clearly shown that although the overall increasing trend is captured in MRC1 and MRC2, the ACE value on the plate surface is greatly underestimated, especially those at the first 4 rows. This is a result of the not well-resolved coolant jet separation and reattachment in the region close to the wall that has been discussed previously. Moreover, the local cell refinement in the wake of the coolant jets, as well as on the surface, improves the prediction of coolant jet reattachment to the plate surface. This is even more important in hybrid LES-RANS because the grid resolution need to be small enough to capture the vortices in the separation and reattachment regions. The results of MRC3 present a similar coolant film coverage as that of the measurements. For the first three rows, the surface ACE after the coolant exit is slightly lower than in the experiment. This is due to the underpredicted amount of coolant flow reattaching to the surface. From the 4th row, an increasing amount of coolant is reattached to the plate surface and the surface ACE increases significantly. Also, the overall temperature of the coolant film consisted of multiple coolant jets is reducing as more coolant flow is injected. This consequentially results in a reduction in the heat transfer rate between the injected coolant flow and the flow in the coolant film region. The two phenomena together result in an increase in the surface ACE distribution. However, the effects may be slightly under-resolved in MRC3. Such underestimate leads to a slightly lower value in the high ACE region near the centreline downstream each coolant hole at $x>25 D$ comparing to that in the experiment. It is noted that the results of the measurements are slightly angled, which is mainly due to a slightly asymmetric geometry of the coolant holes when the plate is manufactured [15, 16].

The spanwise-averaged mean ACE profiles are plotted in Fig. 14. An area-weighted spanwiseaveraging process is used, which includes cells from regions inside the coolant hole where the ACE value is about 1.0. This results in the occurrence of peaks in the ACE profiles with an interval of about 6.2D, which is the distance between two rows of coolant holes, as Fig.14(a) illustrates. The spanwise-averaged mean ACE profile presented in [15] is obtained with a slightly different averaging process. Therefore, the spanwise-averaging process for the simulation results is modified to obtain profiles as close to the measurements as possible, and is shown in Fig. 14 (b). Encouraging results are obtained by MRC3 compared with the measurements and RANS, while results of RANS calculations largely underestimate the surface ACE. The increasing trend of the surface ACE after each hole is captured well by the hybrid LES-RANS approach while the RANS results show a much slower increase. A small underestimate of 


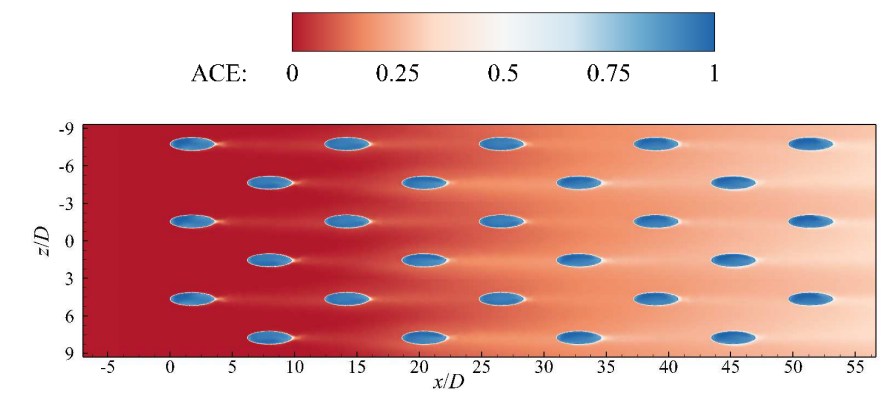

(a) MRC1

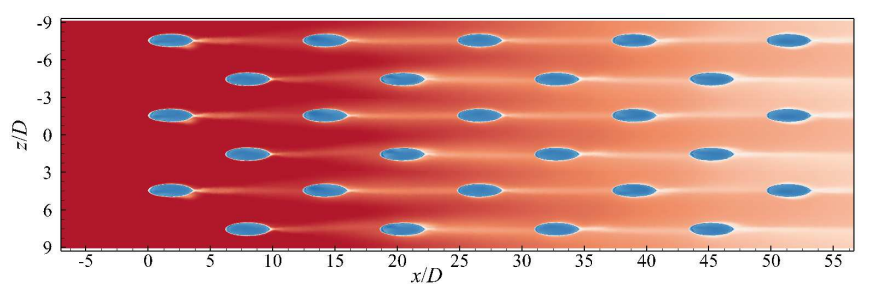

(b) MRC2

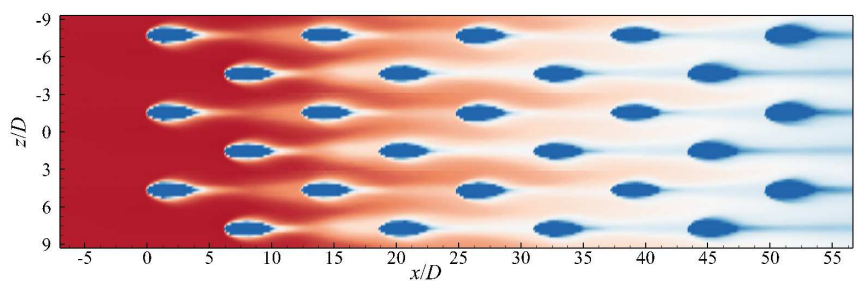

(c) MRC3

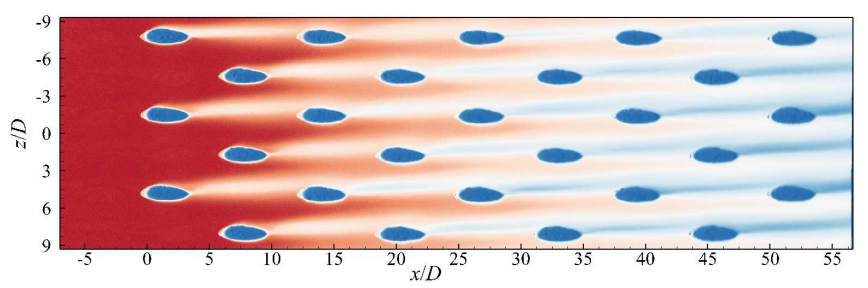

(d) Experiment

Figure 13: Contours of time-averaged surface adiabatic cooling effectiveness: (a) MRC1, (b) MRC2, (c) MRC3

the surface ACE occurs in the downstream region of $x>10 D$ in MRC3. It suggests that the amount of coolant reattaching to the plate surface is slightly underpredicted. This is possibly due to that the local grid resolution is not fine enough to accurately resolve all scales of the turbulent flow structures in the reattachment regions, which consist of the upstream turbulence, coolant reattachment, jet-mainstream mixing etc. These structures, especially those close to the plate surface, bring coolant jets and mainstream together and thus determine the temperature and coverage of the coolant film near the plate surface. Therefore, the extent to which those structures are resolved greatly influences the behaviour of the coolant film and surface ACE. Additionally, the increasing grid resolution introduces improvements on surface ACE prediction over the three cases, although such improvements in RANS solutions is not as great as between RANS and hybrid LES-RANS. 


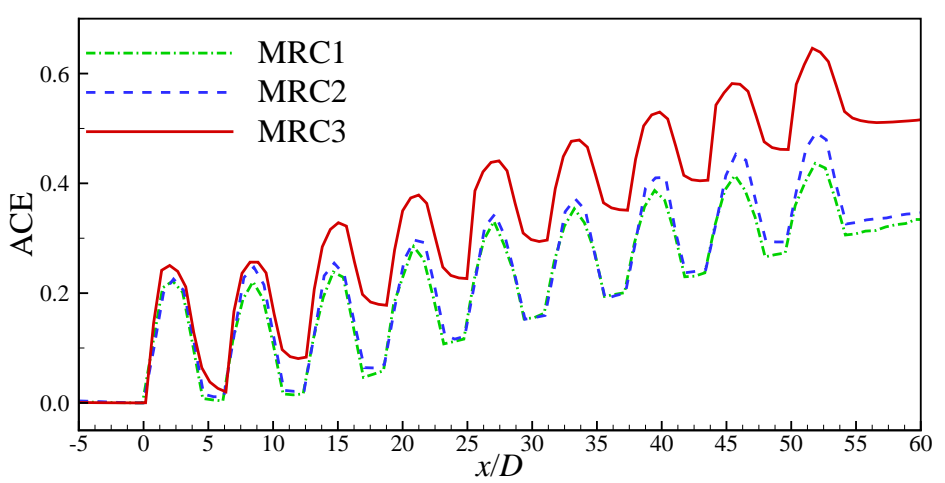

(a) Comparison of simulations

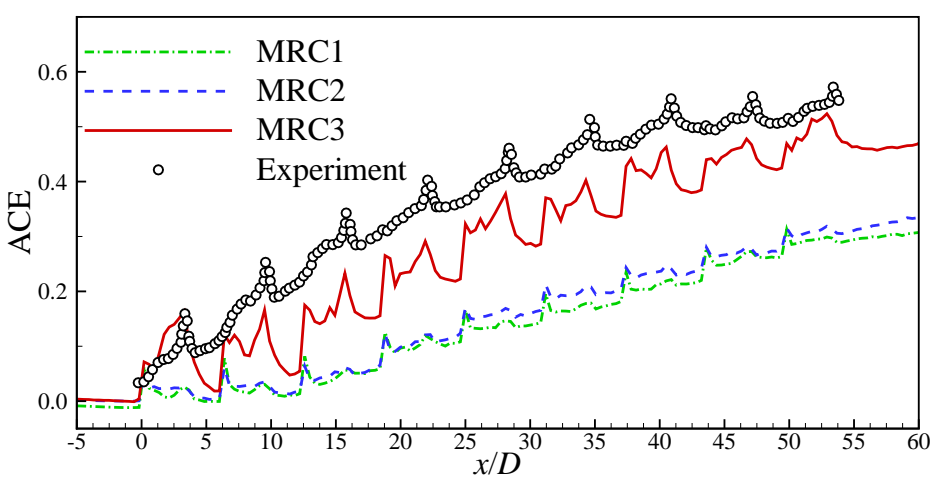

(b) Comparison with measurements

Figure 14: Spanwise-averaged mean surface adiabatic cooling effectiveness: (a) comparison between simulation results , (b) comparison with measurements

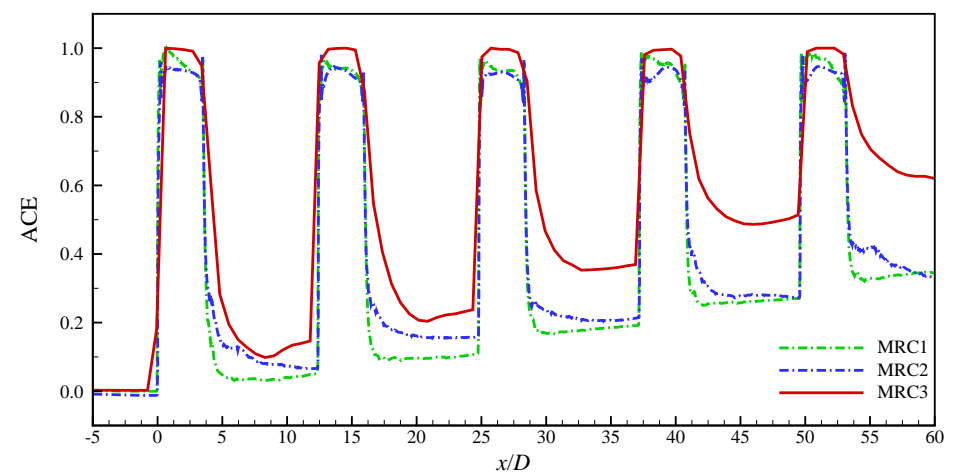

Figure 15: Streamwise distribution of time-averaged surface adiabatic cooling effectiveness through the first column holes at $z=-1.55 D$

The streamwise distribution of the ACE through the centreline of the first coolant hole column is depicted in Fig. 15. Results of MRC3 show an improving prediction of the ACE values in the wake of the downstream coolant holes, though underestimate is found after the first two peaks. Improvements on different grid resolutions is clearly illustrated by the ACE values in the wake regions. Also, the RANS solutions failed to follow the increasing trend closely in the streamwise direction, while this trend is mainly caused by the complex mixing between the mainstream, upstream and downstream coolant jets. It is also suggested that the reattachment and mixing of the initial coolant jets (e.g. first 2 coolant jets 


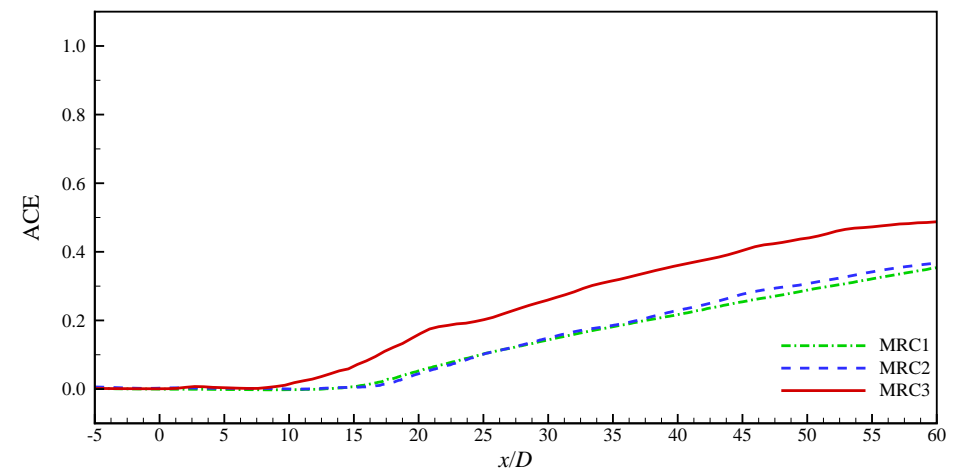

Figure 16: Streamwise distribution of mean surface adiabatic cooling effectiveness at centreline between two columns at $z=0$

in the local column) requires a much finer grid resolution for accurate prediction of the mixing effect.

Fig. 16] illustrates the mean ACE at the centreline between the two column of holes. A constantly increasing trend is shown in all cases. Case MRC3 presents a good prediction of the first non-zero point. This point indicates that the coolant film generated by the two columns of jets started to merge together, and thus provides protection to the plate surface in the middle of the columns. Meanwhile, the first non-zero point in MRC1 and MRC2 is more towards the downstream, and the ACE values are underestimated. The reason that MRC1 and MRC2 give similar prediction along the centreline is that the local grid resolution of the two grids is similar. This suggests that the grid resolution in regions between the two columns of coolant jets is also important, and coarse grid resolution in such regions would result in underestimate of the coolant film spanwise distribution.

\subsection{Coolant film thickness}

As Fig. [4shows, the wakes of the coolant jets form a region where the flow temperature is lower than the mainstream temperature. This region is attached to the plate surface. In this section, it is referred to as the coolant film, since it is a coolant flow region to protect the plate from the high temperature mainstream. The edge of this coolant film can be viewed as the boundary between the coolant jets and the hot mainstream. In order to statistically define the coolant film region, a temperature recovery factor, which is similar to the velocity recovery factor used in the boundary layer [36], is calculated as follows,

$$
F_{T}(\mathbf{x})=\frac{T(\mathbf{x})-T_{c}}{T_{\infty}-T_{c}}
$$

in which $F_{T}(\mathbf{x})$ denotes the temperature recovery factor at a specific point in the flow domain. If $F_{T}=1$, the local temperature fully recovers to the mainstream temperature. If $F_{T}<1$, it is regarded as being inside the coolant film region. In this way, the boundary between the coolant film region and the mainstream can be defined by a given value of temperature recovery factor. The boundaries of the coolant film based on a temperature recovery factor of $F_{T}=99 \%$ are shown in Fig. 17a and an additional picture of boundaries with a lower temperature recovery factor of $F_{T}=75 \%$ is presented by Fig $17 \mathrm{~b}$, It is clear that the coolant film only covers the regions directly downstream the coolant hole in the first 
few rows. As more coolant jets are injected to the mainstream, the spanwise coverage of the coolant film develops until the whole plate surface is isolated from the hot mainstream.

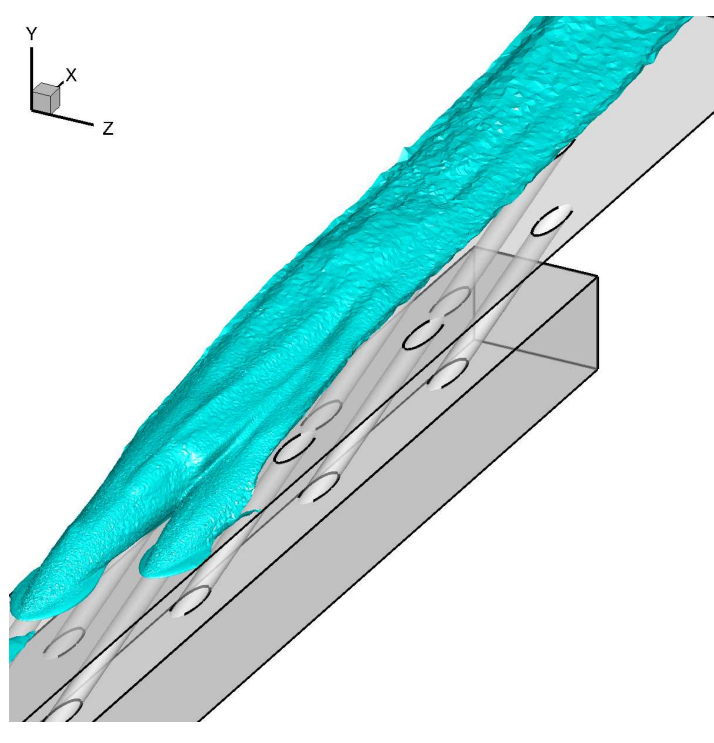

(a) $F_{T}=99 \%$

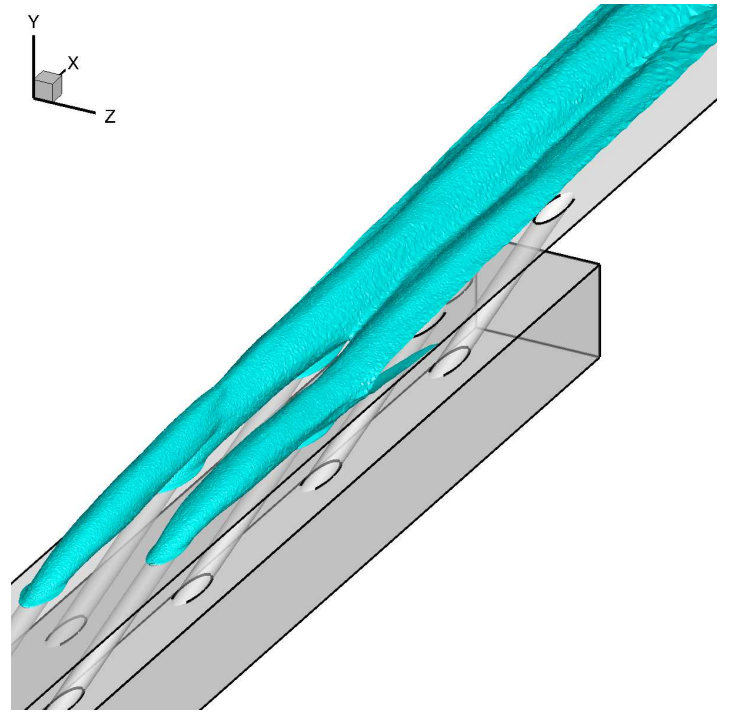

(b) $F_{T}=75 \%$

Figure 17: Isosurfaces of the coolant film of MRC3

In order to quantify the coverage of the coolant film in the spanwise and vertical direction, a coolant film "thickness" can be defined as the vertical distance between the coolant film boundaries to the plate surface. Reminiscent of the velocity boundary layer thickness defined at the position where the velocity recovery factor reaches $99 \%$ [36], the coolant film thickness $\delta_{F_{T}}$ can be defined when the temperature recovery factor reaches $99 \%$,

$$
\delta_{99 \%}=\max \left(F_{T}^{-1}(k=99 \%)\right)
$$

Detailed comparison of the coolant film thickness at different positions and the development in the streamwise and spanwise direction are plotted in Figs. 18 and 19, The profiles suggest that the coolant jets form a consistent protection layer at the position directly downstream the coolant hole, while the plate surface between the two columns is not covered by the coolant until the 3 rd row $(x=10 D)$. It is also shown that the coolant film at the 2nd column is closer to the plate surface due to the staggered arrangement. As a result, the coolant film thickness between the two columns is smaller than the 1st column but greater than the 2 nd column. Additionally, the coolant film thickness increases very fast in the first few rows, which can be regarded as a developing region of the coolant film. Downstream of $x \approx 15 D$, the rate of increase reduces, and a complete protection layer covering the whole spanwise of the plate surface has formed. Such region where $x>15 D$ can be regarded as the developed region of the coolant film.

\subsection{Coolant jet trajectory}

The coolant jet trajectory can be represented by the centre of each coolant jet. In this paper, the temperature difference $F_{T}$ is used to identify the coolant jet centre, and the trajectory is illustrated in 


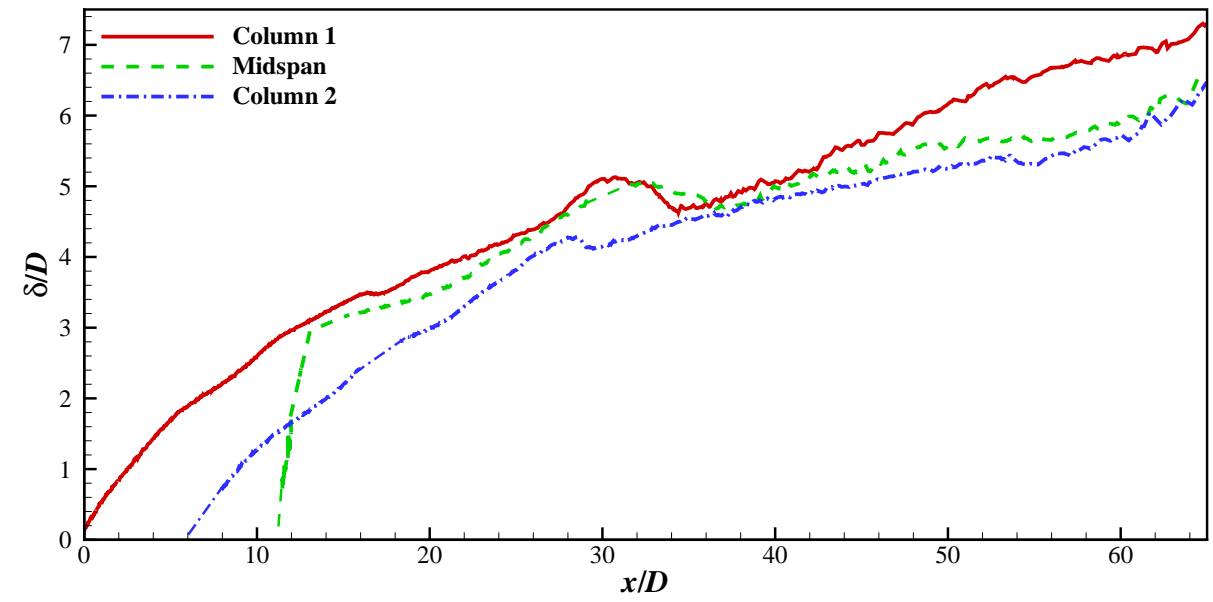

Figure 18: Streamwise coolant film thickness $\delta_{99 \%}$ distribution

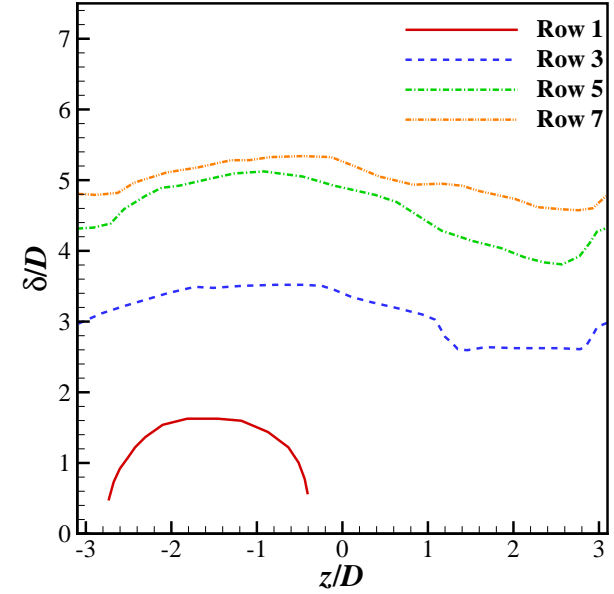

(a) 1st column of holes

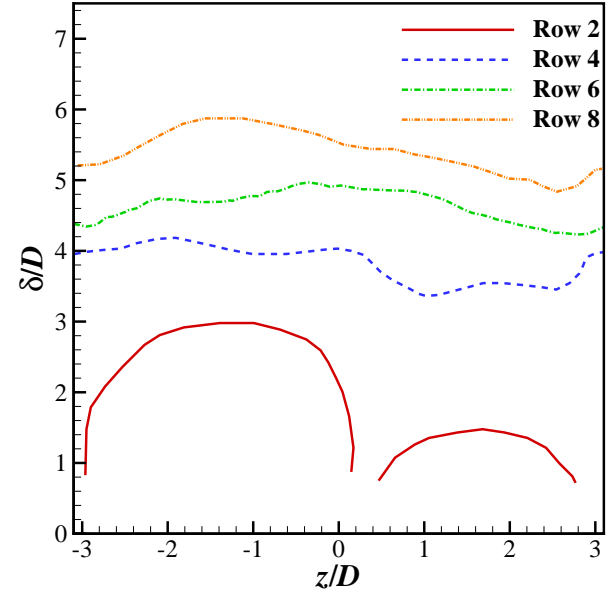

(b) 2nd column of holes

Figure 19: Development of the spanwise coolant film thickness $\delta_{99 \%}$ evaluated at the trailing edge of the 1st and 2nd columns of coolant holes

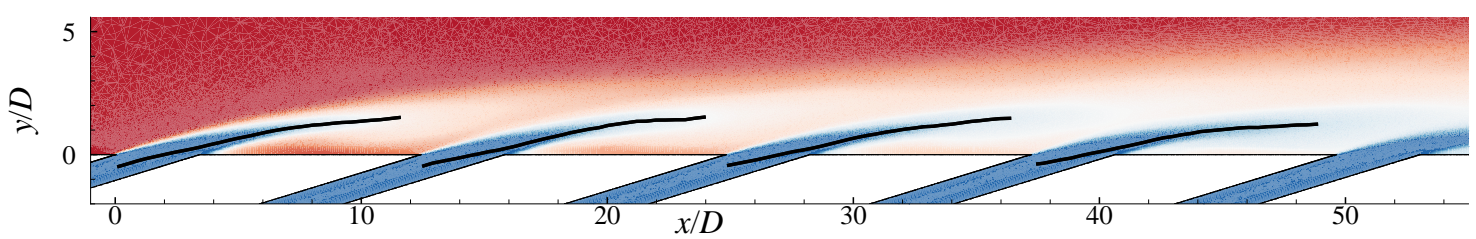

Figure 20: Coolant jet centre trajectory on contours of temperature with diverging blue and red colour

Fig. 20,

The profiles coolant jet trajectory are then compared as Fig. 21 shows. The starting point of the downstream trajectories (jet 3,5 and 7) is aligned with that of the first coolant jet at $x=0$, to demonstrate the different behaviour of coolant jet in the downstream position. A common trend can be found from profiles of the two cases that the first coolant jet in the column are blended towards the plate 
surface due to the mixing with the mainstream. However, the downstream coolant jet, which is the 3rd coolant jet, penetrates further in the vertical direction. This is because that the 1st coolant jet forms a preliminary coolant film that adjust to the coolant velocity and temperature, and this preliminary coolant film reduces the shear stress when the downstream coolant jet injects to the mainstream. However, the limitation on the coolant jet vertical penetration increases when moving further towards the downstream. This restriction is due two reasons. The first reason is the blockage due to more fluid injected into the mainstream that forces the injected jet to stay attached to the plate surface. The second reason is that a complete coolant film is formed towards the downstream with the velocity becomes more towards the direction that is parallel to the plate surface, and the downstream coolant jet is blended once injected to the mixed coolant film.

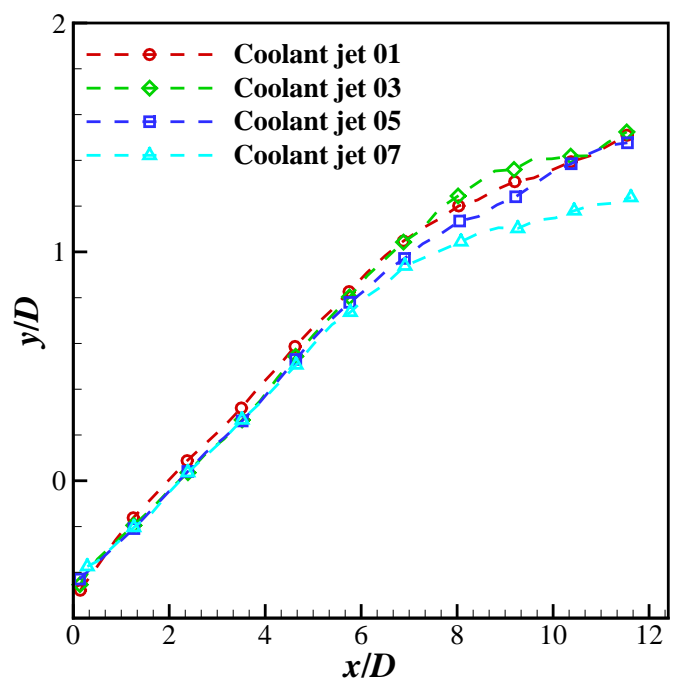

Figure 21: Time-averaged trajectories of coolant jets in the 1st column which is able to predict the mean surface adiabatic cooling effectiveness from given design parameters like injection angle, blowing ratio etc. Part of those models use information of coolant jet trajectory, either measured or predicted, to calculate the corresponding mean ACE distribution. For example, the method proposed by LeGrives et al. 41] involves two mechanisms controlling the ACE: the mass based on the information provided by the coolant jet trajectory, either from measurements or prediction. Although such model is restricted to single-row configuration, study of jet trajectories in multi-row configuration can be beneficial to the development of analytical or semi-analytical models dedicated in the mean ACE prediction of multi-row configurations. 


\section{Turbulent flow structures}

\subsection{Identification of turbulent flow structures}

The instantaneous flow features can be used to study the relation between the turbulent flow structures and the coolant film distribution on the plate surface. The instantaneous three dimensional flow structures of MRC3 are illustrated by isosurfaces of $\lambda_{2}$ at an non-dimensional value of -0.128 , and coloured by temperature in Fig. 22. The $\lambda_{2}$ is the second largest eigenvalue of the velocity gradient tensor, and it is considered one of the best ways to show the vortex core [42]. Shear layers are formed at the interface between the coolant jet from the first hole in each column and the mainstream. The Kelvin-Helmholtz structures can be observed from the leading edge of the first hole in each column. These structures develop and break down into smaller structures in the downstream region. The hairpin vortices are generated due to the interaction with the plate surface. In the multi-row case, vortices in the wake region of the first coolant jet in each column are present until the mixed flow comes across the jet from the next coolant hole. Effects between the upstream and downstream coolant jets occur as discuss previously, and result in the downstream coolant jet being more attached to the plate surface. This phenomenon would reduce the coolant film temperature attached to the surface and therefore increases the surface ACE. It is also known that the effect becomes more dominant when it moves further to the downstream, since more coolant jets join the mixed flow. Also, a wider spanwise spreading of the coolant film is obtained as the downstream coolant jet is more attached to the plate surface.

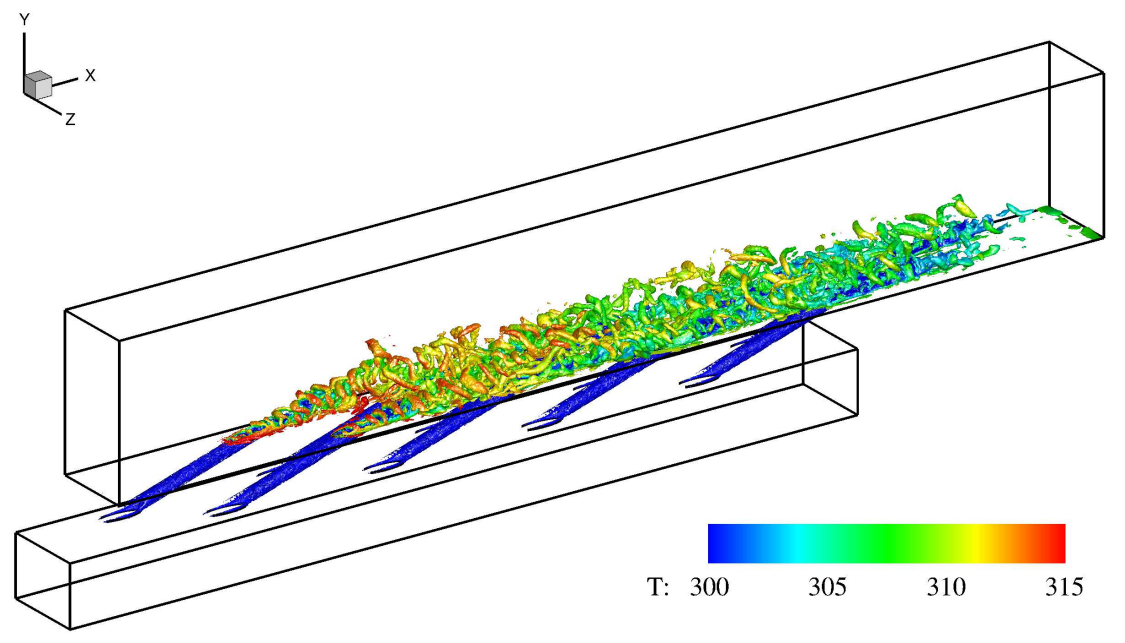

Figure 22: Three-dimensional flow structures depicted by $\lambda_{2}$ and coloured by temperature

The instantaneous temperature contours and spanwise vorticity on a slice through the first column of holes are presented in Fig. 23 and Fig. 24 respectively. The turbulent inflow, jet-mainstream shear layers and smaller structures caused by the separation and reattachment of the coolant flow are clearly illustrated in the contours of vorticity. The effects of these structures on the near-wall flow temperature can be studied from the contours of temperature. As Fig. 23 shows, the shear layer formed at the leading edge of the first hole is broken down by the mainstream turbulence not far after the injection. Hence, the coolant jet penetration into the mainstream is shortened. This leads to a faster reattachment of 
the coolant jet to the plate surface, which would reduce the near-wall flow temperature. However, the inflow turbulence also enhances the mixing of coolant jet and hot mainstream. The combination of the two effects results in the surface ACE downstream the first 2 holes in Fig. 23 remaining at a low level. Moreover, the early breakdown of the coolant jet also leads to reduced effects on the direct downstream coolant jet, which make the downstream coolant jet being more attached to the plate surface. Therefore, the surface ACE value in regions downstream the 2 nd and 3rd coolant holes decreases significantly after the trailing edge due to the fast breakdown of the coolant jets. As illustrated in the surface ACE distribution, the effect that results in the coolant jets being more attached to the plate surface becomes dominant after the 6 th row.

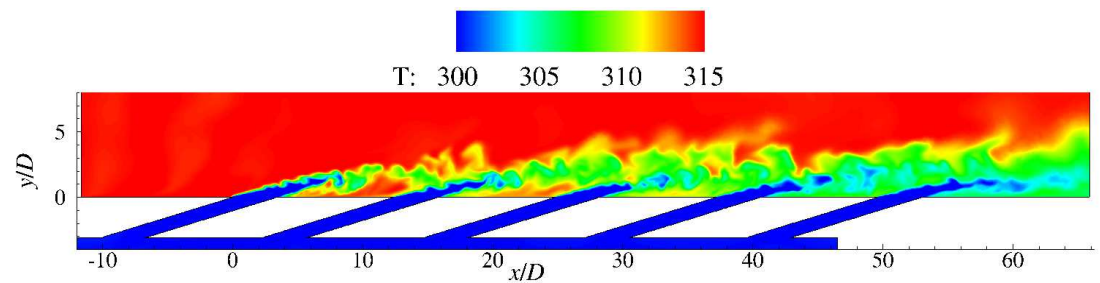

Figure 23: Instantaneous contours of temperature through the first column holes

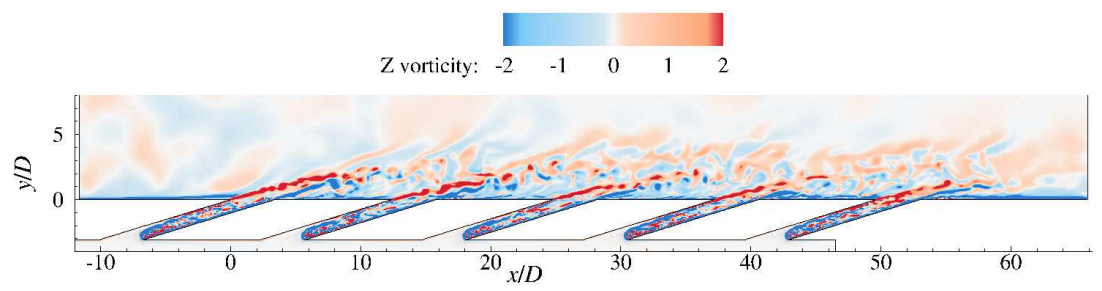

Figure 24: Instantaneous contours of non-dimensional spanwise vorticity through the first column holes

The instantaneous temperature and streamwise vorticity contours at selected downstream positions are shown in Fig. 25] and Fig. 26] respectively. It is found from the vorticity contours that the two shear layers die out soon after the trailing edge of the 1st and 2nd row of holes. Moreover, the coolant jets from the 1st and 2nd coolant holes break up before they reach the downstream hole. This confirms the finding that the inflow turbulence breaks down the coolant jet early. It is also proved by the temperature contours that the effect that results in the coolant jet being more attached to the plate surface becomes more obvious after the 4th row. The inflow turbulence in the mainstream is found to exist throughout the channel. Furthermore, the off-wall structures downstream the first coolant hole vanishes quickly in the streamwise direction. Meanwhile, those structures in the downstream coolant jets stay active further in the streamwise direction. This is contributed to the enhanced reattachment and flow mixing in the off-wall regions of the downstream coolant jets, as they are more attached to the plate surface.

\subsection{Spectral analysis}

The unsteady features of the flow field are also examined by the power spectra in the upper shear layer and on the wall downstream of the first column coolant holes. Fig. 27 shows a sketch of the probe positions to extract the time signal for the spectral analysis. Two groups of probes are considered. The 


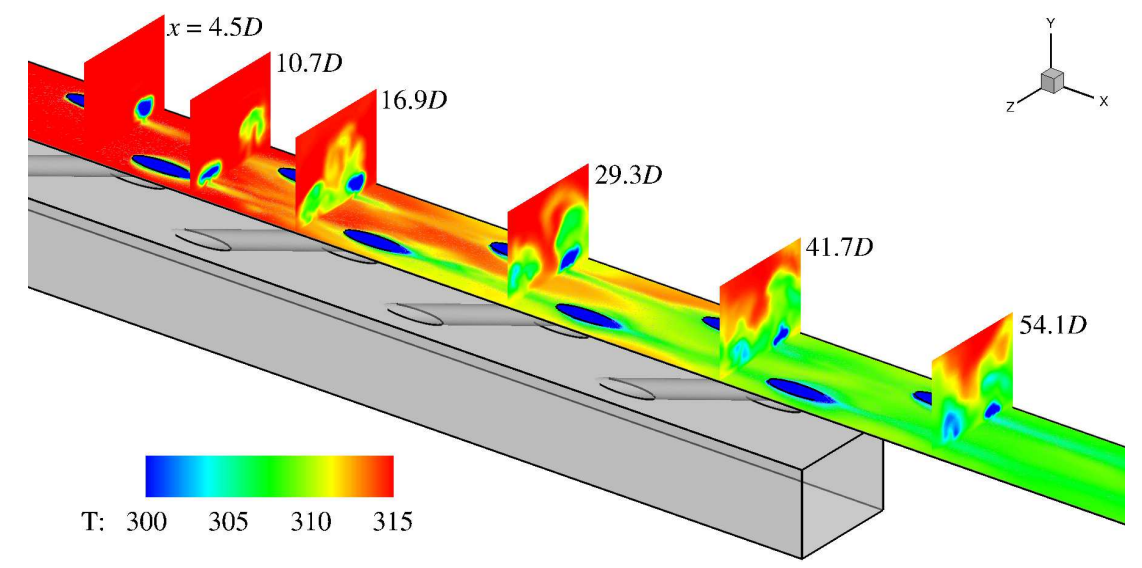

Figure 25: Instantaneous contours of temperature on the plate surface and selected downstream slices

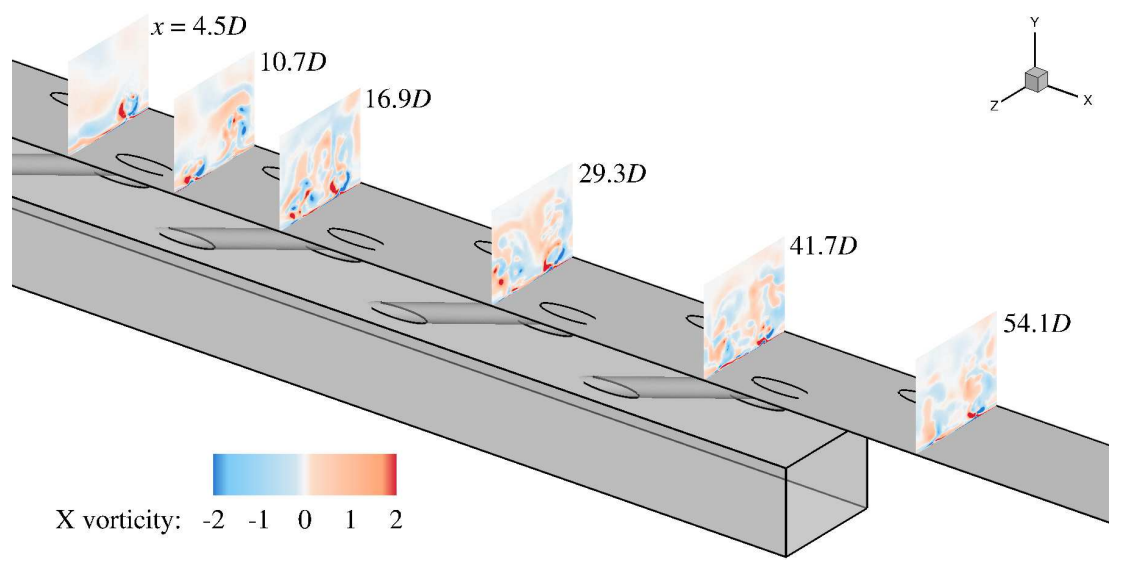

Figure 26: Instantaneous contours of non-dimensional streamwise vorticity on the plate surface and selected downstream slices

first group is located in the upper shear layer $y=1 D$ at the trailing edge of each coolant exit. The second group is placed on the plate surface and at the same streamwise position as the first group.

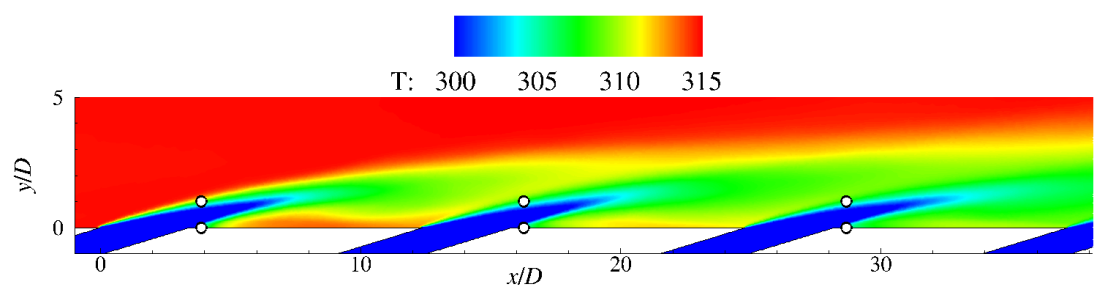

Figure 27: A sketch showing the location of the probes for the spectral analysis

The spectra of turbulence kinetic energy (TKE) and pressure fluctuation in the upper shear layer are shown in Fig. 28. The TKE spectra in the shear layer of all 5 holes follow the Kolmogorov's $-5 / 3$ law in the inertial subrange. It demonstrates that the turbulent flow structures in the upper shear layer is fully developed. A few modes can be found in the spectra, especially from that of the 1st hole. One of the modes is clearly illustrated by the spectra of pressure fluctuation at about $8000 \mathrm{~Hz}$, which corresponds to a Strouhal number of about 0.65 . This frequency is considered to be the major frequency of the shear layer instability. However, this peak diminishes in the spectra of the downstream holes because the small 
turbulent flow structures in mixed flow destroys the potential shear layer that might be formed between the mixed flow and downstream coolant jets.

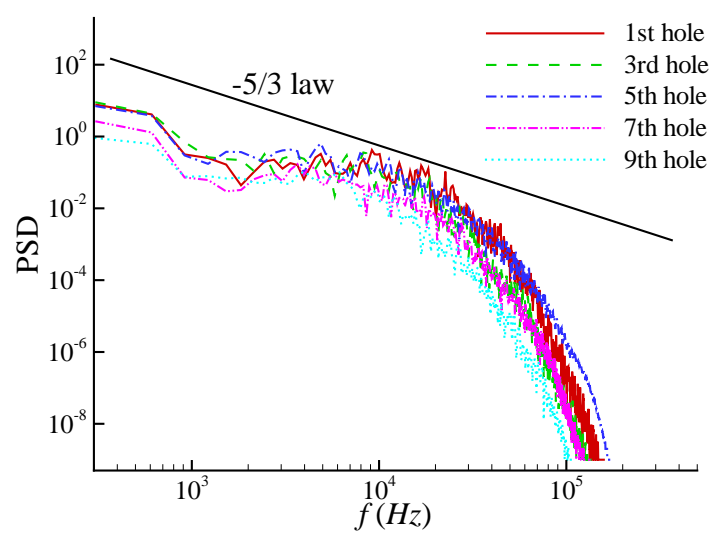

(a) TKE

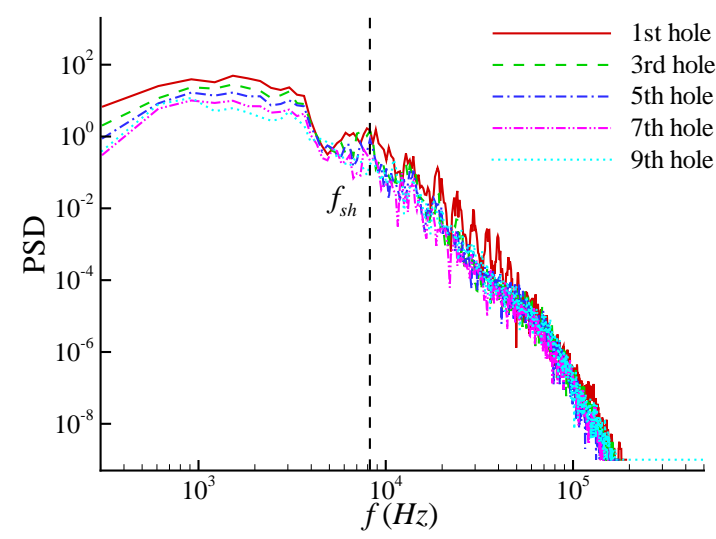

(b) Pressure fluctuation

Figure 28: Power spectral density of (a) turbulence kinetic energy and (b) pressure fluctuation in the shear layer

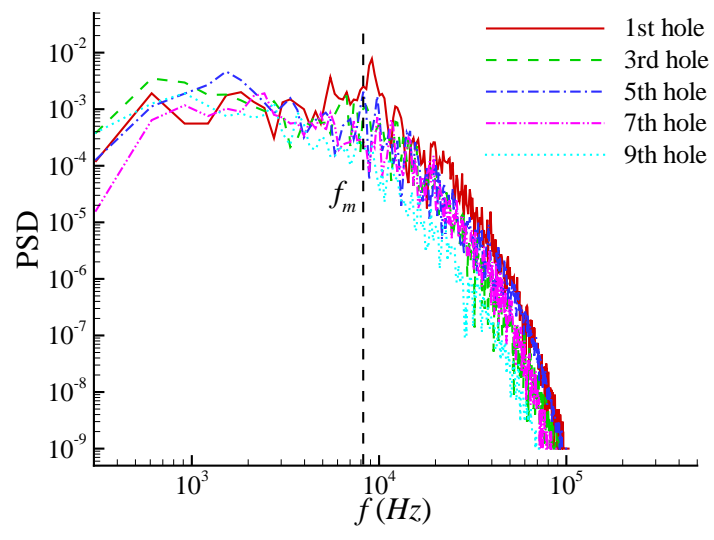

(a) Shear layer

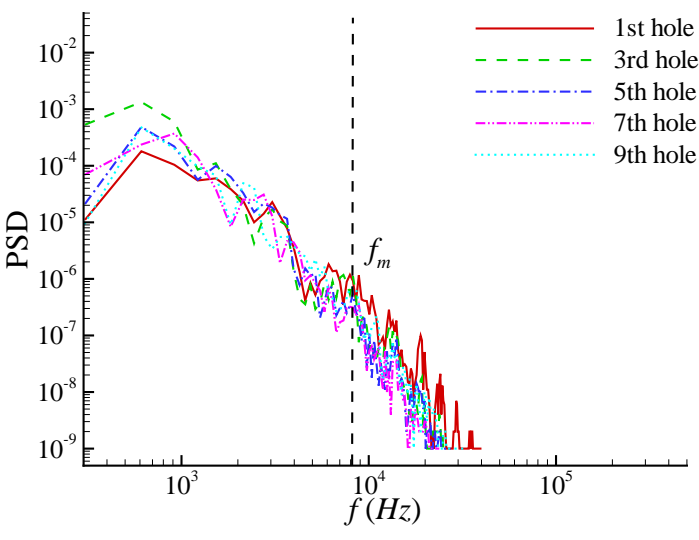

(b) Wall

Figure 29: Power spectral density of temperature fluctuation (a) in the shear layer and (b) on the wall

It is clearly shown that the temperature spectra both in the shear layer and close to the plate surface have a major peak frequency at about $8000 \mathrm{~Hz}$, which is consistent with the shear layer instability frequency. The second and third peaks are also found in the spectra of the first hole both in the upper shear layer and on the wall. This is because the upstream flow for the first coolant jet does not contain any mixed coolant flow. Therefore, the instability in the shear layer is purely generated by the mixing of coolant jet and mainstream. Moreover, the presence of lower shear layer also brings a few frequency modes to the temperature spectrum on the wall. However, the frequency modes diminish in the spectra at downstream positions. This is because the coolant injected to the mainstream from the upstream holes results in a reducing temperature of the mixed flow. Therefore, the temperature fluctuation becomes smaller in the downstream holes are more attached to the plate surface so that the temperature fluctuation is restricted 
and the frequency modes are diminished.

\subsubsection{Spectral coherence}

An attempt to study the similarity of the unsteady features in the wake of the upstream and downstream coolant jets is carried out by using the coherence function between the two time-dependant signals. Following [43], the magnitude-squared coherence function between two temporal signals $a(t)$ and $b(t)$ is calculated from,

$$
\mathscr{R}_{a b}^{2}(f)=\frac{\left|S_{a b}(f)\right|^{2}}{S_{a a}(f) S_{b b}(f)}
$$

where $S_{a b}(f)$ is the cross-spectral density of the signals $a(t)$ and $b(t), S_{a a}(f)$ and $S_{b b}(f)$ are the power spectral density functions of the corresponding signal, and the coherence function $\mathscr{R}_{a b}^{2}(f)$ varies between

0 and 1 . The two signals are perfectly correlated at a certain frequency $f$ if the corresponding coherence function $\mathscr{R}_{a b}^{2}=1$. In this particular case, it suggests that turbulent flow structures with the same size occurs at both the position of $a$ and $b$. On the contrary, the two signals are uncorrelated at a certain frequency $f$ while the corresponding coherence function $\mathscr{R}_{a b}^{2}=0$. The spectral coherence analysis is a common tool in signal post-processing and has been in used various studies such as noise and vibration transmission [44], vibro-acoustics detection [45], electrical impedance measurements [46] etc.

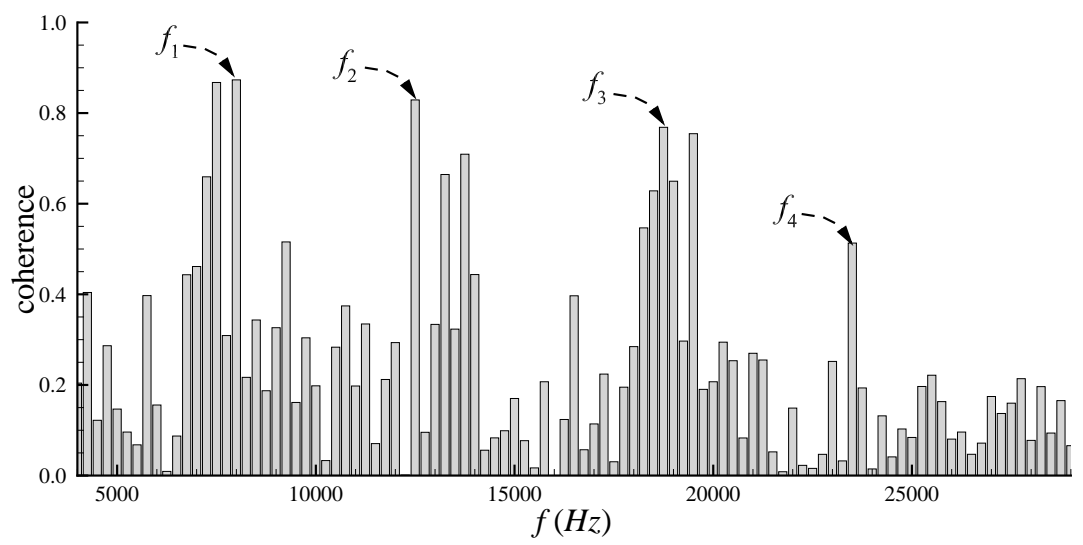

(a) 1st and 3rd hole

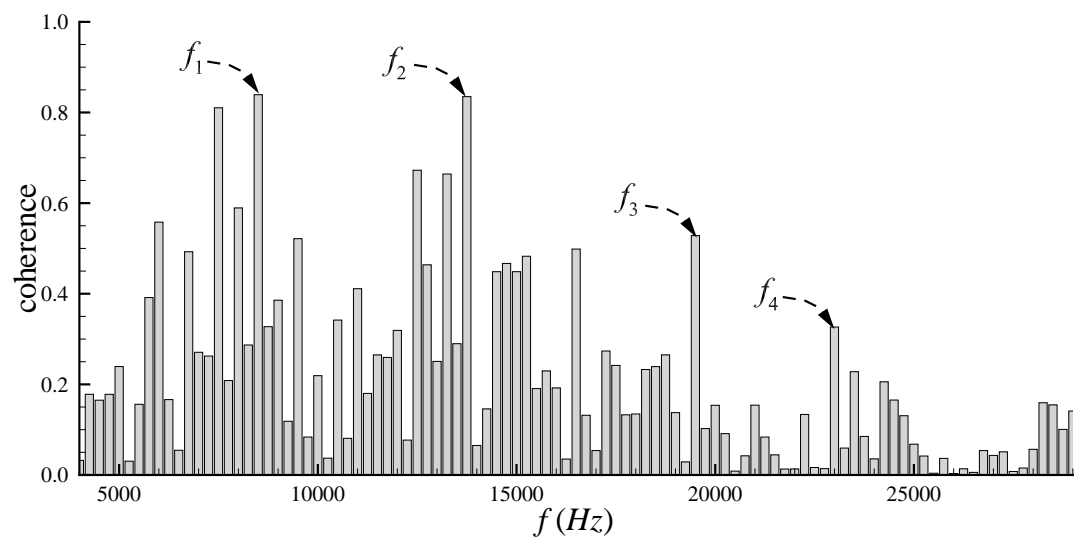

(b) 1st and 7th hole

Figure 30: Coherence function of wall pressure fluctuations between: (a) the 1st and 3rd hole, (b) the 1st and 7th hole 


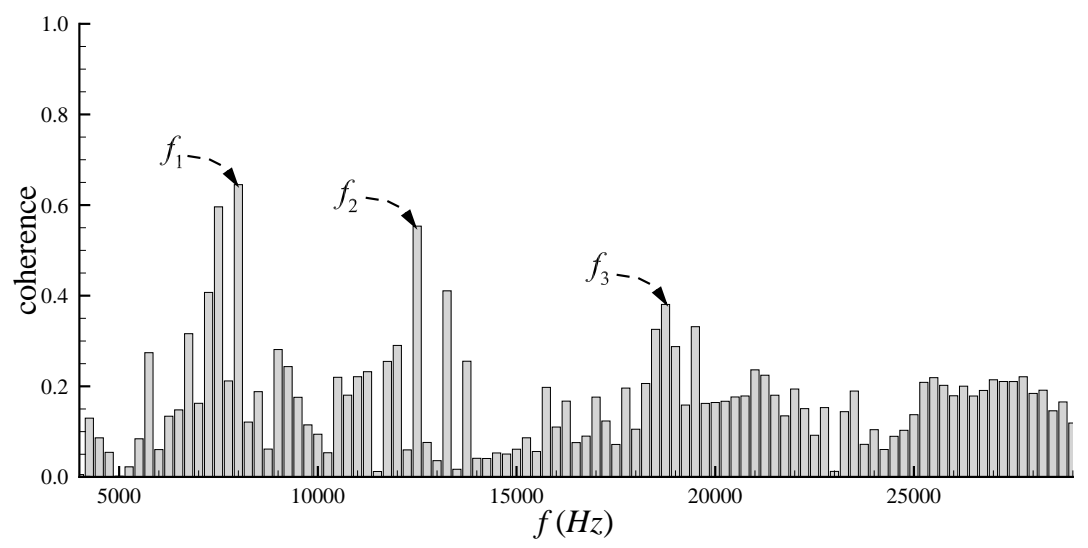

(a) 1st and 3rd hole

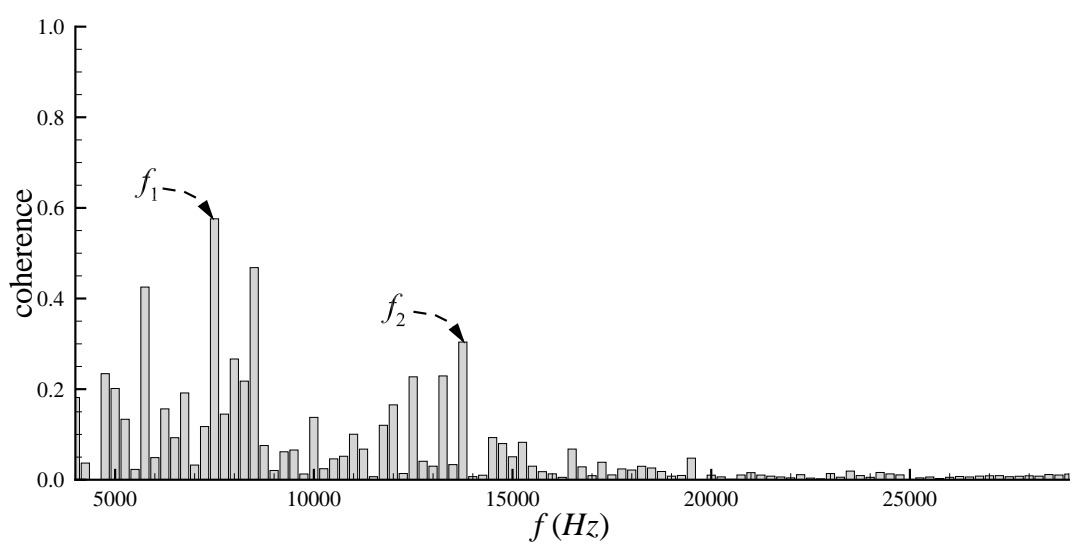

(b) 1st and 7th hole

Figure 31: Coherence function of wall temperature fluctuations between: (a) the 1st and 3rd hole, (b) the 1st and 7th hole

The coherence of the pressure and temperature fluctuations close to the plate surface between the 1st hole and the downstream holes is studied in this section. The aim of calculating coherence of the pressure and temperature signals from the wake of the first row and the downstream rows is to find out the structures that exist both in the upstream and downstream coolant jets, as well as their effects on the hot-cold flow mixing. Fig. 30 illustrates the coherence of pressure fluctuation. Four modes can be found from the pressure coherence between the 1st and 3rd hole. This first peak occurs at a frequency of about $8000 \mathrm{~Hz}$, which is the major frequency of the coolant jet lower shear layer. And this frequency is consistent with the major frequency of the coolant jet upper shear layer. Three higher frequency modes $f_{2}, f_{3}$ and $f_{4}$ can be found close to $13000 \mathrm{~Hz}, 19000 \mathrm{~Hz}$ and $24000 \mathrm{~Hz}$ respectively. The coherence of the 2nd mode keeps high in both Fig. 30 (a) and (b). This suggests that turbulent flow structures corresponding to this frequency keeps occurring in the downstream coolant jets. Meanwhile, coherence of the 3rd and 4th modes reduces to below 0.5 in Fig. 30 (b), which indicates that structures corresponding to the two frequencies are not as active in the wake of 7 th coolant jet.

The coherence function of wall temperature is presented in Fig. 31. Peaks can be found in both profiles near the major frequency of about $8000 \mathrm{~Hz}$. This indicates that the major shear layer roll-up has a great influence on the mixing of coolant jet and mainstream, although it is close to the plate 
surface. Two higher frequency modes occur close to $13000 \mathrm{~Hz}$ and $19000 \mathrm{~Hz}$ corresponding to the 2nd and 3rd modes in the coherence function of wall pressure fluctuation. However, the 3rd mode disappears in Fig. 31 (b), and the value of 2nd mode reduces to below 0.5. It suggests that the effect of turbulent flow structures corresponding to a frequency near $19000 \mathrm{~Hz}$ is very small on hot-cold mixing close to the wall, while the peak close to $13000 \mathrm{~Hz}$ diminishes quickly in the downstream position.

\section{Conclusion}

The performance of a multi-row effusion cooling configuration with cylindrical coolant holes at scaled combustor conditions has been studied numerically in this paper. The time-averaged temperature and velocity distribution across the field are investigated to provide an overview of the mixed flow field formed by the coolant jets and mainstream, as well as the formation of the coolant film. More detailed study on the coolant film distribution and formation is carried out based on the adiabatic cooling effectiveness and analysis of the temperature field. Results suggest that the prediction of coolant film distribution is highly dependent on the resolution of the turbulent flow structures that are important to the coolantmainstream mixing, especially in regions close to the plate surface where part of the coolant jets separate and reattach. The formation of the coolant film across the multi-row array is investigated using a "film thickness" defined based on the temperature recovery factor. It is found that the plate surface directly downstream the coolant holes are well covered by the coolant film, while regions in the middle of the two columns are exposed to the hot mainstream until the 3rd row. Study on the coolant jet trajectories show that the downstream coolant jets are more attached to the plate surface. As a result, the mean ACE and spanwise coverage of the coolant jet is increasing with the streamwise location. The increasing coolant attachment is due to the fact that the upstream coolant jets injected to the mainstream together form a low-temperature and high-velocity coolant film on top of the plate surface, which reduces the blowing ratio locally at the position where the downstream coolant jet is injected.

The flow features and their effects on the coolant film are investigated in the later part of the paper, especially the turbulent flow structures and coolant-mainstream mixing close to the plate surface. A major frequency of the coolant jet shear layer instability is found at about $8000 \mathrm{~Hz}$ in both the upper and lower boundaries. This frequency is found to diminish in the upper shear layer of the downstream coolant jets, which is due to the rapid destruction of the downstream coolant jet shear layer by the turbulent flow structures in the upstream flow. On the contrary, such frequency mode can still be found in the coolant jet lower shear layer close to the plate surface, although the strength is weak. Spectral coherence analysis is carried out on the near-wall pressure and temperature signals of different coolant holes in the 1st column. It is demonstrated that turbulent flow structures from the coolant jet lower shear layer is closely related to the near-wall flow temperature, and can be found in all coolant jets from upstream to downstream.

This study has shown that the plate surface is well protected when the coolant film is formed after 3-4 rows of coolant holes, but the plate surface between the first few rows of coolant holes are still exposed to the hot mainstream. Hence, some extra features should be added to the cooling scheme to protect this region. The results of velocity field, pressure difference between the inlet and exit of the coolant holes and 
coolant massflow distribution can be used in the development and calibration of $1 \mathrm{D}$ and 2D prediction models being used widely in the initial design stage of effusion cooilng schemes. The information of the unsteady flow field, especially the Reynolds stresses, are essential in helping the development of reducedorder models and more accurate RANS models with anisotropic Reynolds stresses or non-linear eddy viscosity. Therefore, results of this study can be helpful in improving the cooling performance and can be beneficial to effusion cooling predictive modelling.

\section{Acknowledgments}

The authors would also like to acknowledge the use of the Hydra High Performance Computing System at Loughborough University. Financial and technical support from the Department of Aeronautical and Automotive Engineering and the Rolls-Royce UTC at Loughborough University made this work possible.

\section{Nomenclature}

\begin{tabular}{|c|c|c|c|c|}
\hline & $\delta_{i j}$ & Kronecer delta & $D R$ & Density Ratio, $\rho_{c} / \rho_{\infty}$ \\
\hline & $\gamma$ & central-upwind blending parameter & $E$ & total energy per unit mass \\
\hline & $\kappa$ & thermal conductivity & $L_{T}$ & turbulent integral lengthscale \\
\hline \multirow[t]{5}{*}{630} & $\kappa_{T}$ & turbulent conductivity & $M$ & Mach number \\
\hline & $\tau$ & stress tensor, $=\left[\tau_{i j}\right]$ & $M R$ & Momentum Ratio, $\rho_{c} U_{c}^{2} / \rho_{\infty} U_{\infty}^{2}$ \\
\hline & $\mathbf{F}$ & inviscid flux vector, $=\left[\mathbf{F}_{i}\right]$ & $N u$ & Nusselt number, $N u=h L / k_{\text {fluid }}$ \\
\hline & G & viscous flux vector, $=\left[\mathbf{G}_{i}\right]$ & $p$ & static pressure of the fluid \\
\hline & Q & vector of primitive variables & $\operatorname{Pr}$ & Prandtl number \\
\hline \multirow[t]{5}{*}{635} & q & heat flux vector, $=\left[q_{i}\right]$ & $\operatorname{Pr}_{T}$ & turbulent Prandtl number \\
\hline & $\mathbf{u}$ & Cartesian components of velocity, $=\left[u_{i}\right]=$ & $R$ & gas constant \\
\hline & & {$[u, v, w]^{T}$} & $R e$ & Reynolds number, $R e=\rho U L / \mu$ \\
\hline & W & vector of conservative variables & $S_{i j}$ & strain-rate tensor \\
\hline & $\mathrm{x}$ & Cartesian coordinates, $=\left[x_{i}\right]=[x, y, z]_{660}^{T_{60}}$ & St & Strouhal number, $S t=f L / U$ \\
\hline \multirow[t]{5}{*}{640} & $\mu$ & viscosity & $T$ & temperature \\
\hline & $\mu_{T}$ & RANS eddy viscosity & $t$ & physical time \\
\hline & $\mu_{s g s}$ & Sub-grid scale eddy viscosity & $t^{*}$ & non-dimensional time unit \\
\hline & $\rho$ & fluid density & $T_{c}$ & coolant flow temperature \\
\hline & $B i$ & Biot number, $B i=h L / k_{\text {solid }}$ & $T_{\infty}$ & mainstream temperature \\
\hline \multirow[t]{3}{*}{645} & $B R$ & Blowing Ratio, $\rho_{c} U_{c} / \rho_{\infty} U_{\infty}$ & $T u$ & turbulence intensity \\
\hline & $c_{p}$ & specific heat & $U_{c}$ & coolant flow bulk velocity at exit \\
\hline & $D$ & diameter of the coolant hole & $U_{\infty}$ & mainstream velocity \\
\hline
\end{tabular}




\section{References}

[13] M. Konopka, W. Jessen, M. Meinke, W. Schröder, Large-eddy simulation of film cooling in an adverse pressure gradient flow, Journal of Turbomachinery 135 (3) (2013) 031031.

[14] D. Martin, Effects of high intensity, large-scale free-stream turbulence on combustor effusion cooling,

[1] A. K. Sinha, D. G. Bogard, M. E. Crawford, Film-cooling effectiveness downstream of a single row of holes with variable density ratio, Journal of Turbomachinery 113 (3) (1991) 442-449.

[2] J. R. Pietrzyk, D. G. Bogard, M. E. Crawford, Effects of density ratio on the hydrodynamics of film cooling, Journal of Turbomachinery 112 (1990) 437.

[3] R. Goldstein, E. Eckert, F. Burggraf, Effects of hole geometry and density on three-dimensional film cooling, International Journal of Heat and Mass Transfer 17 (1974) 595-607.

[4] R. Goldstein, T. Yoshida, The influence of a laminar boundary layer and laminar injection on film cooling performance, Journal of Heat Transfer 104 (2) (1974) 355-362.

[5] D. K. Walters, J. H. Leylek, A systematic computational methodology applied to a three-dimensional film-cooling flowfield, Journal of Turbomachinery 119 (4) (1997) 777-785.

[6] A. Azzi, B. Jubran, Numerical modeling of film cooling from short length stream-wise injection holes, Heat and Mass Transfer 39 (2003) 345-353.

[7] Y. C. Li, C. M. Cheng, Y. L. Lo, F. M. Fang, D. Q. Zheng, Simulation of turbulent flows around a prism in suburban terrain inflow based on random flow generation method simulation, Journal of Wind Engineering and Industrial Aerodynamics 146 (2015) 51-58.

[8] M. Tyagi, S. Acharya, Large eddy simulation of film cooling flow from an inclined cylindrical jet, Journal of Turbomachinery 125 (2004) 737-742.

[9] K. Liu, R. Pletcher, Large eddy simulation of discrete-hole film cooling in a flat plate turbulent boundary layer, in: 38th AIAA Thermo-physics Conference, American Institute of Aeronautics and Astronautics, 2005, pp. AIAA paper No. 2005-4944.

[10] P. Renze, W. Schröder, M. Meinke, Large-eddy simulation of film cooling flows with variable density jets, Flow, Turbulence and Combustion 80 (1) (2008) 119-132.

[11] J. Ziefle, L. Kleiser, Numerical investigation of a film-cooling flow structure: Effect of crossflow turbulence, Journal of Turbomachinery 135 (4) (2013) 041001:1-12.

[12] E. Motheau, T. Lederlin, J. L. Florenciano, P. Bruel, LES investigation of the flow through an effusion-cooled aeronautical combustor model, Flow, turbulence and combustion 88 (1) (2012) 169189.

Ph.D. thesis, Loughborough University, Loughborough, UK (2013). 
[15] J. Krawciw, D. Martin, P. Denman, Measurement and prediction of adiabatic film effectiveness of combustor representative effusion arrays, in: ASME Turbo Expo 2015: Turbine Technical Conference and Exposition, American Society of Mechanical Engineers, 2015, pp. V05BT12A035V05BT12A035.

[16] J. Krawciw, Optimisation techniques for combustor wall cooling, Ph.D. thesis, Loughborough University, Loughborough, UK (2017).

[17] A. Travin, M. L. Shur, M. Strelets, P. R. Spalart, Detached-eddy simulations past a circular cylinder, Flow, Turbulence and Combustion 63 (1) (2000) 293-313. doi:10.1023/A:1009901401183.

[18] H. Xia, P. G. Tucker, Numerical simulation of single-stream jets from a serrated nozzle, Flow, Turbulence and Combustion 88 (2012) 3-18. doi:10.1007/s10494-011-9377-5.

[19] X. Chen, H. Xia, A hybrid LES-RANS study on square cylinder unsteady heat transfer, International Journal of Heat and Mass Transfer 108 (2017) 1237-1254. doi:10.1016/j.ijheatmasstransfer.2016.10.081.

[20] H. Xia, Dynamic grid detach-eddy simulation for synthetic jet flows, Ph.D. thesis, The University of Sheffield, Sheffield, UK (2005).

[21] H. Xia, P. G. Tucker, S. Eastwood, Large-eddy simulations of chevron jet flows with noise predictions, International Journal of Heat and Fluid Flow 30 (6) (2009) 1067-1079. doi:10.1016/j.ijheatfluidflow.2009.05.002.

[22] L. Davison, S. H. Peng, Hybrid LES-RANS modelling: A one-equation SGS model combined with a $k-\omega$ model for predicting recirculating flows, International Journal for Numerical Methods in Fluids 43 (9) (2003) 1003-1018. doi:10.1002/fld.512.

[23] P. R. Spalart, W. H. Jou, M. Strelets, S. R. Allmaras, Comments on the feasibility of LES for wings, and on a hybrid RANS/LES approach, Advances in DNS/LES 1 (1997) 4-8.

[24] M. L. Shur, P. R. Spalart, M. Strelets, A. Travin, Detached-eddy simulation of an airfoil at high angle of attack, in: W. Rodi, D. Laurence (Eds.), Engineering Turbulence Modelling and Experiments 4, Elsevier Science Ltd., 1999, pp. 669-678.

[25] F. R. Menter, M. Kuntz, R. Langtry, Ten years of industrial experience with the SST turbulence model, in: K. Hanjalic, Y. Nagano, M. Tummers (Eds.), Turbulence, Heat and Mass Transfer 4, Begell House Inc., 2003, pp. 625-632.

[26] J. Smagorinsky, General circulation experiments with the primitive equations: I the basic equations, Monthly Weather Review 91 (3) (1963) 99-164.

[27] F. Nicoud, H. B. Toda, O. Cabrit, S. Bose, J. Lee, Using singular values to build a subgrid-scale model for large eddy simulations, Physics of Fluids 23 (8) (2011) 085106. 
[28] M. Strelets, Detached eddy simulation of massively separated flows, in: 39th Aerospace sciences meeting and exhibit, 2001, p. 879.

[29] J. Boris, F. Grinstein, E. Oran, R. Kolbe, New insights into large eddy simulation, Fluid Dynamics Research 10 (4-6) (1992) 199.

[30] L. Margolin, W. Rider, F. Grinstein, Modelling turbulent flow with implicit LES, Journal of Turbulence 7 (15) (2006) 1-27.

[31] K. Ritos, I. W. Kokkinakis, D. Drikakis, S. M. Spottswood, Implicit large eddy simulation of acoustic loading in supersonic turbulent boundary layers, Physics of Fluids 29 (4) (2017) 046101. doi:10.1063/1.4979965

[32] S. Pope, Turbulent flows, Cambridge University Press, Cambridge, 2000.

[33] N. Jarrin, Synthetic inflow boundary conditions for the numerical simulation of turbulence, Ph.D. thesis, University of Manchester, Manchester, UK (2008).

[34] R. Poletto, T. Craft, A. Revell, A new divergence free synthetic eddy method for the reproduction of inlet flow conditions for LES, Flow, Turbulence and Combustion 91 (3) (2013) 519-539.

[35] A. Skillen, A. Revell, T. Craft, Accuracy and efficiency improvements in synthetic eddy methods., International Journal of Heat and Fluid Flow 62 (2016) 386-394.

[36] H. Schlichting, K. Gersten, Boundary Layer Theory, Springer, 2016.

[37] R. J. Goldstein, Film cooling, Advances in Heat Transfer 7 (1971) 321-379.

[38] B. Lakshminarayana, Fluid Dynamics and Heat Transfer of Turbomachinery, Wiley-Interscience publication, John Wiley \& Sons, 1995.

URL https://books.google.co.uk/books?id=v6EvNjt0JcoC

[39] S. Baldauf, A. Schulz, S. Wittig, M. Scheurlen, An overall correlation of film cooling effectiveness from one row of holes, in: ASME 1997 International Gas Turbine and Aeroengine Congress and Exhibition, American Society of Mechanical Engineers, 1997, pp. V003T09A010-V003T09A010.

[40] E. Le Grives, Vorticity associated with the penetration of a jet into a cross flow, Journal of Engineering for Power JULY 100 (3) (1978) 465.

[41] E. Le Grives, Cooling techniques for modern gas turbines, Chapter 4 in Topics in Turbomachinery Technology (D. Japikse, ed.), Concepts ETI, Inc., Norwich, VT, 1986.

[42] J. Jeong, F. Hussain, On the identification of a vortex, Journal of Fluid Mechanics 285 (1995) 69-94. doi:10.1017/S0022112095000462.

[43] K. Shin, J. Hammond, Fundamentals of signal processing for sound and vibration engineers, John Wiley \& Sons, 2008. 
[44] A. G. González, J. Rodrıguez, X. Sagartzazu, A. Schumacher, I. Isasa, Multiple coherence method in time domain for the analysis of the transmission paths of noise and vibrations with non stationary signals, Proceedings of ISMA 2010.

[45] J. M. Muggleton, M. J. Brennan, Y. Gao, Determining the location of buried plastic water pipes from measurements of ground surface vibration, Journal of Applied Geophysics 75 (1) (2011) 54 61.

[46] R. A. Nazer, V. Cattin, P. Granjon, M. Montaru, M. Ranieri, Broadband identification of battery electrical impedance for hevs, IEEE Transactions on Vehicular Technology 62 (7) (2013) 2896-2905. doi:10.1109/TVT.2013.2254140.

[47] Y. H. Choi, C. L. Merkle, The application of preconditioning in viscous flows, Journal of Computational Physics 105 (2) (1993) 207-223.

[48] F. R. Menter, Improved two-equation $k$ - $\omega$ turbulence models for aerodynamic flows, Tech. Rep. TM 103975, NASA (1992).

\section{Appendix A. The preconditioning of the governing equations}

The governing equations are transformed to their primitive form for the simplicity of the Jacobian. The primitive form not only brings advantages in the analysis of the preconditioning but also simplifies the numerical solving procedure, particularly for the implicit scheme of the viscous terms [47].

$$
\frac{\partial \mathbf{W}}{\partial \mathbf{Q}} \frac{\partial}{\partial t} \int_{\Omega} \mathbf{Q} d V+\oint_{\partial \Omega}(\mathbf{F}-\mathbf{G}) \cdot \mathbf{n} d A=0
$$

where the primitive variables $\mathbf{Q}=\left[p, u_{i}, T\right]^{T}$, and the Jacobian $\partial \mathbf{W} / \partial \mathbf{Q}$ is used to convert conservative variables $\mathbf{W}$ to primitive variables $\mathbf{Q}$.

Time-derivative preconditioning is implemented to overcome numerical stiffness at low Mach conditions. This is achieved by replacing the density derivative $\rho_{p}$ in the Jacobian $\partial \mathbf{W} / \partial \mathbf{Q}$ with the preconditioning variable $\Theta$, which is a function of Mach number.

In this way, the time-derivative preconditioning system of Navier-Stokes equations can be written as,

$$
\boldsymbol{\Gamma} \frac{\partial}{\partial t} \int_{\Omega} \mathbf{Q} d V+\oint_{\partial \Omega}(\mathbf{F}-\mathbf{G}) \cdot \mathbf{n} d A=0
$$

where $\Gamma$ is the modified Jacobian,

$$
\boldsymbol{\Gamma}=\left[\begin{array}{ccccc}
\Theta & 0 & 0 & 0 & \rho_{T} \\
\Theta u & \rho & 0 & 0 & \rho_{T} u \\
\Theta v & 0 & \rho & 0 & \rho_{T} v \\
\Theta w & 0 & 0 & \rho & \rho_{T} w \\
\Theta H-1 & \rho u & \rho v & \rho w & \rho_{T} H+\rho c_{p}
\end{array}\right]
$$

More details of the preconditioning variable $\Theta$ and the modified Jacobian $\Gamma$ are presented in [20]. 

for steady flows. To extend its capability to unsteady problems, a pseudo time term is introduced and the original time-derivatives are kept, which reads,

$$
\frac{\partial}{\partial t} \int_{\Omega} \mathbf{W} d V+\Gamma \frac{\partial}{\partial \tau} \int_{\Omega} \mathbf{Q} d V+\oint_{\partial \Omega}(\mathbf{F}-\mathbf{G}) \cdot \mathbf{n} d A=0
$$

where the first term on the left hand side is the physical time term, the second term is the pseudo time denoted by $\tau$ and preconditioning is only applied to this term. The physical time term vanishes for steady state problems, and in unsteady problems the above equation is suitable for the dual-time algorithm.

\section{Appendix B. Transport equations of the SST model for hybrid LES-RANS}

In the two-equation SST $k$ - $\omega$ model [25], the eddy viscosity $\mu_{T}$ is defined as,

$$
\mu_{T}=\frac{\bar{\rho} \alpha_{1} k}{\max \left(\alpha_{1} \omega, \Omega F_{2}\right)}
$$

in which the turbulent kinetic energy $k$ and specific dissipation rate $\omega$ satisfy two transport equations,

$$
\begin{gathered}
\frac{\partial k}{\partial t}+\frac{\partial \widetilde{u}_{i} k}{\partial x_{i}}=\widetilde{P}_{k}-B_{k}+\frac{\partial}{\partial x_{i}}\left[\left(\nu+\sigma_{k} \nu_{T}\right) \frac{\partial k}{\partial x_{i}}\right] \\
\frac{\partial \omega}{\partial t}+\frac{\partial \widetilde{u}_{i} \omega}{\partial x_{i}}=\left(\frac{\alpha}{\nu_{t}}\right) \widetilde{P}_{k}-\beta \omega^{2}+\frac{\partial}{\partial x_{i}}\left[\left(\nu+\sigma_{\omega} \nu_{T}\right) \frac{\partial \omega}{\partial x_{i}}\right]+2\left(1-F_{1}\right) \frac{\sigma_{\omega_{2}}}{\omega} \frac{\partial k}{\partial x_{i}} \frac{\partial \omega}{\partial x_{i}}
\end{gathered}
$$

where $\nu_{T}=\mu_{T} / \bar{\rho}$ is the kinematic eddy viscosity, all coefficients and functions such as $\widetilde{P}_{k}, \Omega, F_{1}, F_{2}, \alpha$, $\alpha_{1}, \beta, \beta^{*}, \sigma_{k}, \sigma_{\omega}, \sigma_{\omega_{2}}$ follow the definition in [25]. It should be noted that the "destruction" term $B_{k}$ in Eq. B.2 is modified as,

$$
B_{k}=k^{3 / 2} \frac{1}{L_{T}} \frac{d}{\widetilde{d}}, \quad L_{T}=\frac{\sqrt{k}}{\beta^{*} \omega}
$$

in which the wall proximity fraction $\widetilde{d} / d$ is used to control the RANS turbulent viscosity $\mu_{T}$, so that the RANS region is blended smoothly with the LES region in the viscosity field.

The SST $k-\omega$ model is well-known for combining the accuracy of Wilcox's $k-\omega$ model in resolving the near wall boundary layer and the low free-stream sensitivity of the Jones-Launder $k-\varepsilon$ model. Additionally, Bradshaw's assumption of the principal shear-stress is considered for extra ability to solve flows with adverse pressure gradients [48]. 